TRANSACTIONS OF THE

AMERICAN MATHEMATICAL SOCIETY

Volume 358, Number 4, Pages 1511-1535

S $0002-9947(05) 03874-2$

Article electronically published on October 31, 2005

\title{
LOCAL THETA CORRESPONDENCE FOR SMALL UNITARY GROUPS
}

\author{
SHU-YEN PAN
}

\begin{abstract}
In this paper we give an explicit parameterization of the local theta correspondence of supercuspidal representations for the reductive dual pairs $\left(\mathrm{U}_{1}(F), \mathrm{U}_{1}(F)\right),\left(\mathrm{U}_{1}(F), \mathrm{U}_{1,1}(F)\right),\left(\mathrm{U}_{1}(F), \mathrm{U}_{2}(F)\right)$, and $\left(\mathrm{U}_{1}(F), \mathrm{U}_{1,2}(F)\right)$ of unitary groups over a nonarchimedean local field $F$ of odd residue characteristic.
\end{abstract}

\section{INTRODUCTION}

Let $F$ be a $p$-adic field with odd residue characteristic. Let $\left(G, G^{\prime}\right)$ be a reductive dual pair over $F$ in the symplectic group $\operatorname{Sp}(W)$. Let $\widetilde{\operatorname{Sp}(W)}, \widetilde{G}$ and $\widetilde{G^{\prime}}$ denote the metaplectic covers of $\operatorname{Sp}(W), G$ and $G^{\prime}$, respectively. By restricting the Weil representation of $\widetilde{\operatorname{Sp}(W)}$ we obtain a correspondence between some irreducible admissible representations of $\widetilde{G}$ and some irreducible admissible representations of $\widetilde{G^{\prime}}$. This correspondence is called the theta correspondence or Howe duality. It is known that the correspondence is one-to-one by R. Howe and J.-P. Waldspurger ( $c f$. MVW87] and Wal90]). Suppose that both the covers $\widetilde{G}$ and $\widetilde{G^{\prime}}$ split ( $c f$. Kud94]). By choosing splittings $G \rightarrow \widetilde{G}$ and $G^{\prime} \rightarrow \widetilde{G^{\prime}}$ we have a one-to-one correspondence between some irreducible admissible representations of $G$ and some irreducible admissible representations of $G^{\prime}$. The fundamental problem is to investigate the explicit theta correspondence. That is, for a given irreducible admissible representation $\pi$ of $G$ we want to understand the following two questions:

1. In what condition of $G^{\prime}$ does $\pi$ occur in the theta correspondence?

2. Which representation $\pi^{\prime}$ of $G^{\prime}$ is $\pi$ paired with once $\pi$ occurs?

The answers of the above two questions are only known for very few examples (among some other partial results), such as $\left(\mathrm{O}_{2}(F), \mathrm{Sp}_{2}(F)\right)$ by J. Shalika and S. Tanaka in ST69 and $\left(\mathrm{U}_{1}(F), \mathrm{U}_{1}(F)\right)$ by C. Moen in Moe93 and by T. Yang in Yan98. In this paper we answer the two questions of when $G$ is $\mathrm{U}_{1}(F)$ and the representation $\pi^{\prime}$ paired with $\pi$ is supercuspidal.

Let $V$ (resp. $V^{\prime}$ ) be a Hermitian (resp. skew-Hermitian) space over a quadratic extension $E$ of $F$. Let $\left(G, G^{\prime}\right)$ be the dual pair $\left(U(V), U\left(V^{\prime}\right)\right)$. In Pan02a we prove that the depths of the paired irreducible admissible representations are equal. Moreover, we prove in $\mathrm{Pan02b}$ and $\mathrm{Pan} 03$ that the minimal $K$-types of the paired representations correspond by theta correspondence of certain finite reductive dual

Received by the editors March 15, 2004.

2000 Mathematics Subject Classification. Primary 22E50; Secondary 11F27, 22E35.

This project was supported by NSC-grant 91-2119-M-006-019 of Taiwan.

(C)2005 American Mathematical Society 
pairs when the depth is zero and by certain orbit correspondence when the depth is positive. Although these results are far from sufficient for determining the explicit correspondence for general reductive dual pairs, they do provide enough information for some special cases; in particular, the cases that are considered in this work. The basic idea of this work is quite simple and can be described as follows. We assume that $V$ is one-dimensional throughout the paper. We use the result in [Pan03] to obtain a decomposition of the space $V^{\prime}$. Then we reduce the problem to the case of correspondence for $\left(\mathrm{U}_{1}(F), \mathrm{U}_{1}(F)\right)$, which is already well known. That means we obtain the information of the minimal $K$-types of $\pi^{\prime}$. Because $\pi^{\prime}$ is supercuspidal, we can conclude that $\pi^{\prime}$ is induced from its minimal $K$-type by the result of Adler in Ad198. Therefore, the representation $\pi^{\prime}$ is determined explicitly.

The contents of this paper are as follows. In section 2 we recall some basic results from Pan02b] and Pan03. In sections 3 and 4 we study the case when both $V$ and $V^{\prime}$ are one-dimensional. This is the easiest case but also the most important case. Of course, the explicit theta correspondence for this dual pair is known and has been studied by several authors, for example, C. Moen (Moe93]) and T. Yang (Yan98). However, we still treat this case completely to expose our point of view. The major difference between our approach and theirs is the use of different models of the Weil representation. We use a generalized lattice model of the Weil representation, while a Schrödinger model is used in Moe93 and a lattice model with respect to a self-dual lattice is used in Yan98. The major part of the paper is section 5. In this section we study the case when $V^{\prime}$ is over an unramified quadratic extension of $F$ and is two-dimensional isotropic. The spirit of our approach is similar to that of Kud86 in some sense. In section 6 we study the case when the quadratic extension of $F$ is ramified. In sections 7,9 and 11 we study the correspondence when $\mathcal{V}^{\prime}$ is over an unramified quadratic extension of $F$ and is two-dimensional anisotropic, three-dimensional and four-dimensional with Witt index one, respectively. In sections 8,10 and 12 we study the parallel cases when $V^{\prime}$ is over a ramified quadratic extension of $F$. The approach is very similar to that used in subsection 5 , so it is very sketchy in these sections. Some partial results of the explicit theta correspondence for the dual pair when $V^{\prime}$ is three-dimensional is also studied by C. Moen in [Moe87] by a different method. It might be interesting to compare his approach and the one used in this paper. In the last section we summarize our result on the first occurrence in previous sections and show that it is consistent with the preservation principle conjectured by S. Kudla and S. Rallis ( $c f$. [HKS96]).

The author thanks the National Science Council of the Taiwan Government for their financial support.

\section{LOCAL THETA CORRESPONDENCE AND Minimal $K$-TYPES}

2.1. Basic notation. Let $F$ be a nonarchimedean local field, $\mathcal{O}_{F}$ the ring of integers of $F, \mathfrak{p}_{F}$ the unique maximal ideal of $\mathcal{O}_{F}, \varpi_{F}$ a uniformizer of $\mathcal{O}_{F}$, and $\mathbf{f}_{F}:=\mathcal{O}_{F} / \mathfrak{p}_{F}$ the residue field. We assume that the characteristic of $\mathbf{f}_{F}$ is odd. Let $q$ denote the cardinality of $\mathbf{f}_{F}$. Let $E$ be a quadratic extension of $F, \mathcal{O}_{E}$ the ring of integers of $E, \mathfrak{p}_{E}$ the unique maximal ideal of $\mathcal{O}_{E}, \varpi_{E}$ a uniformizer of $\mathcal{O}_{E}, \mathbf{f}_{E}$ the residue field of $E$, and $\tau$ the non-trivial automorphism of $E$ over $F$. We assume that $\varpi_{E}=\varpi_{F}$ if $E$ is a unramified extension, and $\tau\left(\varpi_{E}\right)=-\varpi_{E}$ if $E$ is ramified over $F$. Let $e$ denote the ramification index of $E$ over $F$. We normalize the discrete 
valuation of $E$ such that the value group of $E^{\times}$is $\frac{1}{e} \mathbb{Z}$. Let ord: $E^{\times} \rightarrow \frac{1}{e} \mathbb{Z}$ denote the discrete valuation.

Let $(V,\langle\rangle$,$) be a (finite-dimensional) nondegenerate \epsilon$-Hermitian space over $E$, where $\epsilon$ is 1 or -1 . Let $G:=U(V)$ be the group of isometries of $(V,\langle\rangle$,$) , i.e., U(V)$ is a unitary group. Let $\kappa$ be a fixed integer associated to $(V,\langle\rangle$,$) . Suppose that L$ is a lattice in $V$. We define

$$
L^{*}:=\left\{v \in V \mid\langle v, l\rangle \in \mathfrak{p}_{E}^{\kappa} \text { for all } l \in L\right\} .
$$

Then $L^{*}$ is also a lattice in $V$ and is called the dual lattice of $L$. A lattice $L$ is called a good lattice if $L^{*} \varpi_{E} \subseteq L \subseteq L^{*}$.

2.2. Local theta correspondence. Let $(V,\langle\rangle$,$) (resp. (V,\langle\rangle)$,$) be an \epsilon$-Hermitian (resp. $\epsilon^{\prime}$-Hermitian) space over $E$ such that $\epsilon \epsilon^{\prime}=-1$. Let $W:=V \otimes_{E} V^{\prime}$. Define

$$
\langle,\rangle:=\operatorname{Tr}_{E / F}\left(\langle,\rangle \otimes \tau\left(\langle,\rangle^{\prime}\right)\right),
$$

where $\operatorname{Tr}_{E / F}$ is the trace map from $E$ to $F$. We know that $\langle$,$\rangle is a skew-symmetric$ $F$-bilinear form on $W$. The pair $\left(U(V), U\left(V^{\prime}\right)\right)$ is a reductive dual pair of unitary groups in $\operatorname{Sp}(W)$. Let $\kappa$ (resp. $\kappa^{\prime}$ ) be a fixed integer associated to $V$ (resp. $V^{\prime}$ ) as in subsection 2.1. We make the assumption that that $\kappa+\kappa^{\prime}$ is odd if $E$ is a ramified extension of $F$. Define the integer

$$
\lambda_{F}:= \begin{cases}\frac{1}{2}\left(\kappa+\kappa^{\prime}+1\right), & \text { if } E \text { is ramified over } F \\ \kappa+\kappa^{\prime}, & \text { if } E \text { is unramified. }\end{cases}
$$

Let $\psi$ be an (additive) character of $F$ of conductor $\mathfrak{p}_{F}^{\lambda_{F}}$. We assume that the duality of lattices in $W$ is defined with respect to the integer $\lambda_{F}$. Let $\widetilde{\operatorname{Sp}(W)}$ be the metaplectic cover of $\operatorname{Sp}(W)$, let $\left(\omega_{\psi}, \mathcal{S}\right)$ be the Weil representation of $\widetilde{\operatorname{Sp}(W)}$ with respect to the character $\psi$ of $F$, and let $\widetilde{U(V)}$ (resp. $\widetilde{U\left(V^{\prime}\right)}$ ) be the inverse image of $U(V)$ (resp. $U\left(V^{\prime}\right)$ ) in $\widetilde{\operatorname{Sp}(W)}$. Now $\widetilde{U(V)} \widetilde{U\left(V^{\prime}\right)}$ is a subgroup of $\widetilde{\operatorname{Sp}(W)}$ and the map $\widetilde{U(V)} \times \widetilde{U\left(V^{\prime}\right)} \rightarrow \widetilde{U(V) \widetilde{U\left(V^{\prime}\right)}}$ is a group homomorphism. Hence, $\left(\omega_{\psi}, \mathcal{S}\right)$ can be regarded as a representation of $\widetilde{U(V)} \times \widetilde{U\left(V^{\prime}\right)}$. An irreducible admissible representation $(\pi, \mathcal{V})$ of $\widetilde{U(V)}$ is said to correspond to an irreducible admissible representation $\left(\pi^{\prime}, \mathcal{V}^{\prime}\right)$ of $\widetilde{U\left(V^{\prime}\right)}$ if there is a nontrivial $\widetilde{U(V)} \times \widetilde{U\left(V^{\prime}\right)}$-map $\Pi: \omega_{\psi} \rightarrow$ $\pi \otimes \pi^{\prime}$. This establishes a correspondence, called the local theta correspondence or Howe duality for the dual pair $\left(U(V), U\left(V^{\prime}\right)\right)$, between some irreducible admissible representations of $\widetilde{U(V)}$ and some irreducible admissible representations of $\widetilde{U\left(V^{\prime}\right)}$. It has been proved by R. Howe ( $c f$. [MVW87]) and J.-P. Waldspurger ( $c f$. Wal90]) that the local theta correspondence is one-to-one (when the residue characteristic of $F$ is odd). We emphasize that the explicit correspondence depends on the character $\psi$ and the forms $\langle$,$\rangle and \langle,\rangle^{\prime}$.

2.3. Splitting of the metaplectic cover. The reductive dual pair $\left(U(V), U\left(V^{\prime}\right)\right)$ is called split if there exist splittings $\widetilde{\beta}_{V^{\prime}}: U(V) \rightarrow \widetilde{U(V)}$ and $\widetilde{\beta}_{V}: U\left(V^{\prime}\right) \rightarrow \widetilde{U\left(V^{\prime}\right)}$ for the extensions $\widetilde{U(V)} \rightarrow U(V)$ and $\widetilde{U\left(V^{\prime}\right)} \rightarrow U\left(V^{\prime}\right)$, respectively. It is known that the dual pairs of unitary groups always split. By composing the splittings $\widetilde{\beta}_{V^{\prime}}$ and $\widetilde{\beta}_{V}$ we have a correspondence between irreducible admissible representations 
of $U(V)$ and irreducible admissible representations of $U\left(V^{\prime}\right)$. Kudla ([Kud94]) constructs an explicit splitting of the metaplectic groups with respect to the Heisenberg model of the Weil representation. A splitting with respect to a generalized lattice model (see subsection 2.6) of the Weil representation is given in Pan01. It is the splitting used in this paper. More details can be found in later sections.

2.4. Theta correspondence for finite unitary groups. Reductive dual pairs and theta correspondence can be defined over a finite field analogously. However, the correspondence might not be one-to-one. It is known that the theta correspondence for finite reductive dual pairs always split. Now we describe some well-known results of the first correspondence of cuspidal representations for certain dual pairs $\left(U(\mathbf{v}), U\left(\mathbf{v}^{\prime}\right)\right)$ of finite unitary groups $(c f$. [How79]). We shall consider the case when $\mathbf{v}$ is one-dimensional. For convenience, we allow the dimension of $\mathbf{v}^{\prime}$ to be zero. Therefore, we make the following conventions. We define the Weil representation of the symplectic group on a zero-dimensional space to be the trivial representation (of the trivial group). If one of the spaces $\mathbf{v}, \mathbf{v}^{\prime}$ is trivial, then the theta correspondence for the dual pair $\left(U(\mathbf{v}), U\left(\mathbf{v}^{\prime}\right)\right)$ is just the trivial representation corresponding to the trivial representation.

Next we assume that $\mathbf{v}^{\prime}$ is also one-dimensional. In this case, the dimension of the Weil representation is $q$-dimensional. All irreducible representations of $U(\mathbf{v})$ except the trivial one occur in the theta correspondence.

Third, we assume that $\mathbf{v}^{\prime}$ is two-dimensional. Every irreducible representation of $U(\mathbf{v})$ occurs in the theta correspondence. Every representation except the sgn representation corresponds to a $(q-1)$-dimensional irreducible cuspidal representation of $U\left(\mathbf{v}^{\prime}\right)$. The sgn representation corresponds to a $q$-dimensional irreducible representation.

Last, we consider the case that $\mathbf{v}^{\prime}$ is three-dimensional. Every irreducible representation of $U(\mathbf{v})$ occurs in the theta correspondence, but only the sgn representation of $U(\mathbf{v})$ corresponds to irreducible cuspidal representation of $U\left(\mathbf{v}^{\prime}\right)$. The trivial representation of $U(\mathbf{v})$ corresponds to the unique unipotent cuspidal representation of $U\left(\mathbf{v}^{\prime}\right)$, which is of dimension $q^{2}-q$.

2.5. Theta correspondence for finite orthogonal and symplectic groups. In this subsection, let $\mathbf{v}$ be the one-dimensional quadratic space over $\mathbf{f}_{F}$. Now $U(\mathbf{v})$ is a group of two elements. We know that $U(\mathbf{v})$ has two characters, namely the triv character and the sgn character. The triv character of $U(\mathbf{v})$ first occurs in the correspondence when $\mathbf{v}^{\prime}$ is zero-dimensional, and it is paired with the trivial character of $U\left(\mathbf{v}^{\prime}\right)$. The sgn character of $U(\mathbf{v})$ first occurs in the correspondence when $\mathbf{v}^{\prime}$ is two-dimensional, and it is paired with a $\frac{q-1}{2}$-dimensional irreducible cuspidal representation of $U\left(\mathbf{v}^{\prime}\right)$.

2.6. Generalized lattice model of the Weil representation. In this subsection we define the generalized lattice model of the Weil representation. Material in this subsection can be found in Wal90.

Let $\psi$ be the character of $F$ defined as in subsection 2.2 , so $\lambda_{F}$ is the smallest integer such that $\left.\psi\right|_{\mathfrak{p}_{F}^{\lambda_{F}}}$ is trivial. Let $B$ be a good lattice in $W$ (with respect to $\left.\lambda_{F}\right)$, that is, $B^{*} \varpi_{F} \subseteq B \subseteq B^{*}$. Let $\mathbf{b}^{*}$ be the quotient $B^{*} / B$. We know that $\mathbf{b}^{*}$ is a vector space over $\mathbf{f}_{F}$ with a nondegenerate skew-symmetric form $\left\langle\langle,\rangle_{\mathbf{b}^{*}}\right.$ induced from $\langle$,$\rangle . Let \mathrm{H}\left(\mathbf{b}^{*}\right)$ be the Heisenberg group associated to the finite symplectic space 
$\left(\mathbf{b}^{*},\left\langle\langle,\rangle_{\mathbf{b}^{*}}\right)\right.$. Let $\bar{\psi}$ denote the character of $\mathbf{f}_{F}$ defined by $\bar{\psi}\left(\Pi_{\mathcal{O}_{F}}(t)\right):=\psi\left(t \varpi_{F}^{\lambda_{F}-1}\right)$, where $t \in \mathcal{O}_{F}$. Let $\left(\bar{\omega}_{\bar{\psi}}, S\right)$ be the Schrödinger model of the Weil representation of the finite symplectic groups $\operatorname{Sp}\left(\mathbf{b}^{*}\right)$ associated to the data $\left(\mathbf{b}^{*},\left\langle\langle,\rangle_{\mathbf{b}^{*}}, \bar{\psi}\right)\right.$. Although the skew-symmetric form $\left\langle\langle,\rangle_{\mathbf{b}^{*}}\right.$ and character $\bar{\psi}$ depend on the choice of a prime element $\varpi_{F}$, the Weil representation $\bar{\omega}_{\bar{\psi}}$ does not. This can easily be seen from the Schrödinger model. Let $\bar{\rho}_{\bar{\psi}}$ denote the representation of $\mathrm{H}\left(\mathbf{b}^{*}\right)$ corresponding to the character $\bar{\psi}$ on the space $S$. Let $\mathrm{H}\left(B^{*}\right):=B^{*} \times \mathfrak{p}_{F}^{\lambda_{F}-1}$. It is easy to check that $\mathrm{H}\left(B^{*}\right)$ is a subgroup of the Heisenberg group $\mathrm{H}(W)$. We have a reduction homomorphism

$$
\Pi_{\mathrm{H}\left(B^{*}\right)}: \mathrm{H}\left(B^{*}\right) \rightarrow \mathrm{H}\left(\mathbf{b}^{*}\right) \quad \text { by } \quad(b, t) \mapsto\left(\Pi_{B^{*}}(b), \Pi_{\mathcal{O}_{F}}\left(t \varpi_{F}^{1-\lambda_{F}}\right)\right),
$$

where $\Pi_{B^{*}}: B^{*} \rightarrow \mathbf{b}^{*}$ and $\Pi_{\mathcal{O}_{F}}: \mathcal{O}_{F} \rightarrow \mathbf{f}_{F}$. Let $K_{B}$ denote the stabilizer of $B$ in $\operatorname{Sp}(W)$, and let

$$
K_{B}^{\prime}:=\left\{g \in K_{B} \mid(g-1) \cdot B^{*} \subseteq B\right\} .
$$

It is clear that $K_{B}^{\prime}$ is a normal subgroup of $K_{B}$, and $K_{B} / K_{B}^{\prime}$ is isomorphic to $\mathrm{Sp}\left(\mathbf{b}^{*}\right)$. Let $\widetilde{\rho}_{\psi}$ denote the representation of $\mathrm{H}\left(B^{*}\right)$ inflated from $\bar{\rho}_{\bar{\psi}}$ by the projection $\Pi_{\mathrm{H}\left(B^{*}\right)}$, and let $\widetilde{\omega}_{\psi}$ be the representation of $K_{B}$ inflated from $\bar{\omega}_{\bar{\psi}}$ by the projection $K_{B} \rightarrow K_{B} / K_{B}^{\prime}$ and the isomorphism $K_{B} / K_{B}^{\prime} \simeq \operatorname{Sp}\left(\mathbf{b}^{*}\right)$. Let $\mathcal{S}(B)$ denote the space of locally constant, compactly supported maps $f: W \rightarrow S$ such that

$$
f(b+w)=\psi\left(\frac{1}{2}\langle w, b\rangle\right) \widetilde{\rho}_{\psi}(b) \cdot f(w),
$$

for any $w \in W, b \in B^{*}$. Define the action $\rho_{\psi}^{B}$ of $\mathrm{H}(W)$ on $\mathcal{S}(B)$ by

$$
\left(\rho_{\psi}^{B}(w, t) . f\right)\left(w^{\prime}\right)=\psi(t) \psi\left(\frac{1}{2}\left\langle w^{\prime}, w\right\rangle\right) f\left(w^{\prime}+w\right)
$$

for $w^{\prime} \in W$ and $(w, t) \in \mathrm{H}(W)$. For $g \in \operatorname{Sp}(W)$, we define $M[g] \in \operatorname{Aut}(\mathcal{S}(B))$ by

$$
\left(M_{B}[g] . f\right)(w):=\int_{B^{*}} \psi\left(\frac{1}{2}\langle b, w\rangle\right) \widetilde{\rho}_{\psi}\left(b^{-1}\right) \cdot\left(f\left(g^{-1} \cdot(b+w)\right)\right) d b,
$$

where $g \in \operatorname{Sp}(W), f \in \mathcal{S}(B), w \in W$ and $d b$ is a Haar measure on $B^{*}$. We can normalize the Haar measure $d b$ such that

$$
\left(M_{B}[k] . f\right)(w)=\widetilde{\omega}_{\psi}(k) \cdot f\left(k^{-1} \cdot w\right)
$$

for $k \in K_{B}$ and $f \in \mathcal{S}(B)$. This realization of the Weil representation is known as a generalized lattice model. For any union of $B^{*}$-cosets $R$, define

$$
\begin{aligned}
& \mathcal{S}(B)_{R}:=\{f \in \mathcal{S}(B) \mid f \text { has support in } R\}, \\
& \mathcal{S}(B)_{w}:=\mathcal{S}(B)_{B^{*}+w} \text { for } w \in W .
\end{aligned}
$$

2.7. (Unrefined) minimal $K$-types. Let $G:=U(V)$, and let $\mathfrak{g} \subset \operatorname{End}_{E}(V)$ be the Lie algebra of $G$. Let $\mathcal{L}:=\left\{L_{i} \mid i \in \mathbb{Z}\right\}$ be a regular small admissible lattice chain with numerical invariant $\left(n, n_{0}\right)$ in $V(c f$. [Pan02a] $)$. Define $L_{i}^{\sharp}:=L_{-i-n_{0}}^{*}$ for each $i \in \mathbb{Z}$. For every nonnegative integer $d$, we define

$$
\begin{aligned}
G_{\mathcal{L},(d+1) / e n}:=\left\{g \in G \mid(g-1) \cdot L_{i} \subseteq L_{i+d+1},(g-1) \cdot L_{i}^{\sharp} \subseteq L_{i+d+1}^{\sharp} \text { for all } i\right\}, \\
G_{\mathcal{L},(d / e n)^{+}}:=\left\{g \in G \mid(g-1) \cdot L_{i} \subseteq L_{i+d+1}^{\sharp},(g-1) \cdot L_{i}^{\sharp} \subseteq L_{i+d} \text { for all } i\right\}, \\
\mathfrak{g}_{\mathcal{L},(d+1) / e n}:=\left\{X \in \mathfrak{g} \mid X \cdot L_{i} \subseteq L_{i+d+1}, X . L_{i}^{\sharp} \subseteq L_{i+d+1}^{\sharp} \text { for all } i\right\}, \\
\mathfrak{g}_{\mathcal{L},(d / e n)^{+}}:=\left\{X \in \mathfrak{g} \mid X \cdot L_{i} \subseteq L_{i+d+1}^{\sharp}, X \cdot L_{i}^{\sharp} \subseteq L_{i+d} \text { for all } i\right\},
\end{aligned}
$$


where $e$ is the ramification index of $E$ over $F$. Let $\mathfrak{g}_{\mathcal{L},-d / e n}^{*}, \mathfrak{g}_{\mathcal{L},(-d / e n)^{+}}^{*}$ be the filtrations as defined in Pan02a. We know that the quotient $G_{\mathcal{L}, d / e n} / G_{\mathcal{L},(d / e n)^{+}}$ is a finite abelian group if $d>0$. Each character of $G_{\mathcal{L}, d / e n} / G_{\mathcal{L},(d / e n)^{+}}$can be realized as a coset in $\mathfrak{g}_{\mathcal{L}, d / e n} / \mathfrak{g}_{\mathcal{L},(d / e n)^{+}}$. A character $\zeta$ of $G_{\mathcal{L}, d / e n} / G_{\mathcal{L},(d / e n)^{+}}$is called a nondegenerate representation if the coset in $\mathfrak{g}_{\mathcal{L}, d / e n} / \mathfrak{g}_{\mathcal{L},(d / e n)}+$ presenting $\zeta$ contains no nilpotent elements. From results in MP94 and Yu98, we know that for every irreducible admissible representation $(\pi, \mathcal{V})$ of $G$ of positive depth there exists a positive integer $d$ and a regular small admissible lattice chain $\mathcal{L}$ such that the space of fixed points $\mathcal{V}^{G_{\mathcal{L},(d / e n)^{+}}}$is nontrivial and the representation of $G_{\mathcal{L}, d / e n} / G_{\mathcal{L},(d / e n)^{+}}$ on $\mathcal{V}^{G_{\mathcal{L},(d / e n)^{+}}}$contains a nondegenerate representation. Moreover, we know that the depth of $(\pi, \mathcal{V})$ is $\frac{d}{e n}$.

If $L$ is a good lattice in $V$, we define

$$
\begin{aligned}
G_{L} & :=\{g \in U(V) \mid g \cdot L=L\}, \\
G_{L, 0^{+}} & :=\left\{g \in U(V) \mid(g-1) \cdot L^{*} \subseteq L,(g-1) \cdot L \subseteq L^{*} \varpi_{E}\right\} .
\end{aligned}
$$

If an irreducible admissible representation of $G$ is of depth zero, then we know that there exists a good lattice $L$ in $V$ such that $\mathcal{V}^{G_{L, 0^{+}}}$is nontrivial.

\subsection{Local theta correspondence and minimal $K$-types (part I). Let}

$$
\left(G, G^{\prime}\right):=\left(U(V), U\left(V^{\prime}\right)\right)
$$

be a reductive dual pair of unitary groups. Suppose that $L$ is a good lattice in $V$. Then $\mathbf{l}:=L / L \varpi_{E}$ and $\mathbf{l}^{*}:=L^{*} / L$ are vector spaces over $\mathbf{f}_{E}$ with induced $\epsilon$-Hermitian forms. Moreover, we know that $G_{L} / G_{L, 0^{+}} \simeq U(\mathbf{l}) \times U\left(\mathbf{l}^{*}\right)$. Let $G^{\prime}:=$ $U\left(V^{\prime}\right)$ and $L^{\prime}$ be a good lattice in $V^{\prime}$, and let $\mathbf{l}^{\prime}$ and $\mathbf{l}^{\prime *}$ be defined analogously. We know that both $U(\mathbf{l})$ and $U\left(\mathbf{l}^{*}\right)$ are finite unitary groups when $E$ is an unramified extension of $F$. If $E$ is a ramified extension of $F$, then one of $U(\mathbf{l})$ and $U\left(\mathbf{l}^{*}\right)$ is a finite orthogonal group, and the other is a finite symplectic group. It is easy to check that

$$
B=B\left(L, L^{\prime}\right):=L \otimes L^{\prime *}+L^{*} \otimes L^{\prime}
$$

is a good lattice in $\mathcal{W}$. From Pan02b, we know that $\left(U(\mathbf{l}) \times U\left(\mathbf{l}^{*}\right), U\left(\mathbf{l}^{* *}\right) \times U\left(\mathbf{l}^{\prime}\right)\right)$ is a (possibly reducible) finite reductive dual pair in the finite symplectic group $\operatorname{Sp}\left(B^{*} / B\right)$. We remark here that the definition of the finite reductive dual pair is in the generalized sense that one of the two groups is allowed to be trivial. The following results are from $[\mathrm{Pan} 02 \mathrm{~b}]$

Proposition. Let $\left(G, G^{\prime}\right):=\left(U(V), U\left(V^{\prime}\right)\right)$ be a reductive dual pair of two unitary groups. Let $(\pi, \mathcal{V})$ (resp. $\left.\left(\pi^{\prime}, \mathcal{V}^{\prime}\right)\right)$ be an irreducible admissible representation of $\widetilde{U(V)}$ (resp. $\left.\widetilde{U\left(V^{\prime}\right)}\right)$ of depth zero such that $\pi$ and $\pi^{\prime}$ are paired by the local theta correspondence. Then there exist good lattices $L, L^{\prime}$ in $V, V^{\prime}$, respectively, such that $\mathcal{V}^{G_{L, 0^{+}}}, \mathcal{V}^{\prime G_{L^{\prime}, 0^{+}}^{\prime}}$ are nontrivial, and there exists a subrepresentation $\eta$ (resp. $\eta^{\prime}$ ) of

$\mathcal{V}^{G_{L, 0^{+}}}$(resp. $\left.\mathcal{V}^{\prime G_{L^{\prime}, 0^{+}}^{\prime}}\right)$ such that $\eta, \eta^{\prime}$ are paired by the correspondence for the finite dual pair

$$
\left(G_{L} / G_{L, 0^{+}}, G_{L^{\prime}}^{\prime} / G_{L^{\prime}, 0^{+}}^{\prime}\right) .
$$

Corollary. Suppose that $\eta, \eta^{\prime}$ are irreducible cuspidal representations and $\eta \otimes \eta^{\prime}$ is a first occurrence of theta correspondence for the finite reductive dual pair

$$
\left(G_{L} / G_{L, 0^{+}}, G_{L^{\prime}}^{\prime} / G_{L^{\prime}, 0^{+}}^{\prime}\right) .
$$


Then $\pi \otimes \pi^{\prime}$ is a first occurrence for the reductive dual pair $\left(G, G^{\prime}\right)$, where $\pi$ is the irreducible supercuspidal representation $c-\operatorname{Ind}_{G_{L}}^{G} \eta$ of $G$ and $\pi^{\prime}$ is the irreducible supercuspidal representation $c-\operatorname{Ind}_{G_{L^{\prime}}^{\prime}}^{G^{\prime}} \eta^{\prime}$ of $G^{\prime}$.

2.9. Local theta correspondence and minimal $K$-types (part II). Recall that $W:=V \otimes_{E} V^{\prime}$ is an $F$-space. Let $\phi: V \otimes_{E} V^{\prime} \rightarrow \operatorname{Hom}_{E}\left(V, V^{\prime}\right)$ be the isomorphism of $F$-spaces given by $\phi\left(v_{1} \otimes v_{2}\right)(x):=v_{2}\left\langle v_{1}, x\right\rangle$ for $v_{1}, x \in V, v_{2} \in V^{\prime}$. Define the moment map $\mathfrak{M}: W \rightarrow \operatorname{End}_{E}(V)$ by

$$
\mathfrak{M}(w):=-\epsilon \varpi_{F}^{1-\lambda_{F}} \phi^{\prime}(w) \circ \phi(w) .
$$

It is known that the image of the map $\mathfrak{M}$ is in the Lie algebra $\mathfrak{g}$ of $U(V)$. Similarly we define the moment map $\mathfrak{M}^{\prime}: W \rightarrow \mathfrak{g}^{\prime}$ by $w \mapsto-\epsilon^{\prime} \varpi_{F}^{1-\lambda_{F}} \phi(w) \circ \phi^{\prime}(w)$.

Let $\mathcal{L}:=\left\{L_{i} \mid i \in \mathbb{Z}\right\}$ (resp. $\mathcal{L}^{\prime}:=\left\{L_{i}^{\prime} \mid i \in \mathbb{Z}\right\}$ ) be a regular small admissible lattice chain in $V$ (resp. $\left.V^{\prime}\right)$ with numerical invariant $\left(n, n_{0}\right)$ (resp. $\left.\left(n, n_{0}^{\prime}\right)\right)$. Let $d$ be a positive integer such that $n+d+n_{0}+n_{0}^{\prime}$ is even. Define

$$
B\left(\mathcal{L}, \mathcal{L}^{\prime}, \frac{d}{n}\right):=\bigcap_{i+j=\frac{-n-d-n_{0}-n_{0}^{\prime}}{2}} L_{i} \otimes L_{j}^{\prime} .
$$

The major theorem in Pan03 is the following:

Proposition. Let $\left(G, G^{\prime}\right):=\left(U(V), U\left(V^{\prime}\right)\right)$ be a reductive dual pair of orthogonal, symplectic or unitary groups. Let $(\pi, \mathcal{V})\left(\right.$ resp. $\left.\left(\pi^{\prime}, \mathcal{V}^{\prime}\right)\right)$ be an irreducible admissible representation of $\widetilde{U(V)}$ (resp. $\left.\widetilde{U\left(V^{\prime}\right)}\right)$. Suppose that $\pi, \pi^{\prime}$ correspond in the theta correspondence, and the depth of $(\pi, \mathcal{V})$ is positive. Then there exist a positive integer $d$, a regular small admissible lattice chain $\mathcal{L}$ (resp. $\left.\mathcal{L}^{\prime}\right)$ in $V$ (resp. $\left.V^{\prime}\right)$ of period $n$ and an element $w \in B\left(\mathcal{L}, \mathcal{L}^{\prime}, \frac{d}{n}\right)$ such that $\left(G_{\mathcal{L}, d / n}, \mathfrak{M}(w)+\mathfrak{g}_{\mathcal{L},(-d / n)^{+}}\right)$ $\left(\right.$ resp. $\left.\left(G_{\mathcal{L}^{\prime}, d / n}^{\prime}, \mathfrak{M}^{\prime}(w)+\mathfrak{g}_{\mathcal{L}^{\prime},(-d / n)^{+}}^{\prime}\right)\right)$ is a minimal $K$-type of $(\pi, \mathcal{V})\left(\operatorname{resp} .\left(\pi^{\prime}, \mathcal{V}^{\prime}\right)\right)$.

\section{Theta CORRESPONDENCE FOR $\left(\mathrm{U}_{1}(F), \mathrm{U}_{1}(F)\right) \mathrm{I}$}

In this section, we assume that $E$ is an unramified quadratic extension of $F$ (i.e., $e=1$ ), and we assume that both $V, V^{\prime}$ are one-dimensional. Let $G:=U(V)$ and $G^{\prime}:=U\left(V^{\prime}\right)$ be the unitary groups. Note that both $G, G^{\prime}$ are compact.

3.1. Lattice chains in $V^{\prime}$. We assume that $V$ is a Hermitian space with the form denoted by $\langle$,$\rangle , and we assume that V^{\prime}$ is a skew-Hermitian space with the form $\langle,\rangle^{\prime}$ defined by $\langle,\rangle^{\prime}:=\delta\langle$,$\rangle , where \delta$ is a number in $E^{\times}$such that $\tau(\delta)=-\delta$. For simplicity we shall assume that $\operatorname{ord}(\delta)=0$. Let $\lambda_{F}, \kappa, \kappa^{\prime}$ be given as in subsection 2.2. Now we have $\lambda_{F}=\kappa+\kappa^{\prime}$ because $E$ is an unramified extension of $F$.

Let $v_{0}$ be an element in $V$ such that $\left\langle v_{0}, v_{0}\right\rangle=1$. Clearly such an element $v_{0}$ exists. Let $L$ be the lattice $v_{0} \mathfrak{p}_{E}^{\lceil\kappa / 2\rceil}$ in $V$, where $\left\lceil\frac{\kappa}{2}\right\rceil$ stands for the largest integer not greater than $\frac{\kappa}{2}$. Clearly $L^{*}=v_{0} \mathfrak{p}_{E}^{\lfloor\kappa / 2\rfloor}$, where $\left\lfloor\frac{\kappa}{2}\right\rfloor$ stands for the smallest integer not less than $\frac{\kappa}{2}$. Hence, $L$ is a good lattice in $V$. Moreover, $L$ is self-dual if $\kappa$ is even, and $L=L^{*} \varpi_{E}$ if $\kappa$ is odd. It is also clear that $L$ is the unique good lattice in $V$. Now we have $G=G_{L}$ and

$$
G_{L, 0^{+}}=\left\{g \in G \mid(g-1) \cdot L \subseteq L \varpi_{E}\right\} .
$$

Let $\mathbf{l}, \mathbf{l}^{*}$ be defined as in subsection 2.8. Now one of $\mathbf{l}, \mathbf{l}^{*}$ is trivial and the other is one-dimensional. We also know that $\mathbf{f}_{E}$ is a quadratic extension of $\mathbf{f}_{F}$ because $E$ is unramified. Hence, we know that $G_{L} / G_{L, 0^{+}}$is isomorphic to $\mathrm{U}_{1}\left(\mathbf{f}_{F}\right) \otimes \mathrm{U}_{0}\left(\mathbf{f}_{F}\right)$. 
Define $L_{i}:=L \varpi_{E}^{i}$ for each $i \in \mathbb{Z}$. It is clear that the lattice chain $\mathcal{L}:=\left\{L_{i} \mid\right.$ $i \in \mathbb{Z}\}$ is the unique regular small admissible lattice chain in $V$. It is easy to check that the numerical invariant $\left(n, n_{0}\right)(c f$. Pan02a $)$ of $\mathcal{L}$ is $(1,0)$ if $\kappa$ is even, and $\left(n, n_{0}\right)=(1,1)$ if $\kappa$ is odd. Therefore, we have

$$
G_{\mathcal{L}, d / 1}=G_{\mathcal{L}, d}=\left\{g \in G \mid(g-1) . L \subseteq L \varpi_{E}^{d}\right\}
$$

for any positive integer $d$. Note that $G_{\mathcal{L}, d} / G_{\mathcal{L}, d^{+}}$is isomorphic to $\mathbf{f}_{F}$. Therefore, the possible depths of irreducible representations of $G$ are all nonnegative integers.

Everything in the previous paragraph can be define analogously for $U\left(V^{\prime}\right)$. Let $v_{0}^{\prime}$ be an element in $V^{\prime}$ such that $\left\langle v_{0}^{\prime}, v_{0}^{\prime}\right\rangle^{\prime}=\delta$. Then $L^{\prime}:=v_{0}^{\prime} \mathfrak{p}_{E}^{\left\lceil\kappa^{\prime} / 2\right\rceil}$ is the unique good lattice in $V^{\prime}$. We have $G_{L^{\prime}}^{\prime} / G_{L^{\prime}, 0^{+}}^{\prime} \simeq \mathrm{U}_{1}\left(\mathbf{f}_{F}\right) \otimes \mathrm{U}_{0}\left(\mathbf{f}_{F}\right)$. The lattice chain $\mathcal{L}^{\prime}:=\left\{L_{i}^{\prime} \mid i \in \mathbb{Z}\right\}$, where $L_{i}^{\prime}:=L^{\prime} \varpi_{E}^{i}$ is the unique regular small admissible lattice chain in $V^{\prime}$. Let $\left(n^{\prime}, n_{0}^{\prime}\right)$ denote the numerical invariant of $\mathcal{L}^{\prime}$. We have $\left(n^{\prime}, n_{0}^{\prime}\right)=(1,0)$ if $\kappa^{\prime}$ is even, and $\left(n^{\prime}, n_{0}^{\prime}\right)=(1,1)$ if $\kappa^{\prime}$ is odd.

3.2. The correspondence. The correspondence for this dual pair $\left(U(V), U\left(V^{\prime}\right)\right)$ is very simple because both $U(V)$ and $U\left(V^{\prime}\right)$ have the same image when embedded as subgroups of $\operatorname{Sp}(W)$. So the correspondence can be regarded as identical (cf. Moe93] and Yan98]). Our viewpoint here is slightly different. We regard one of the groups $U(V), U\left(V^{\prime}\right)$ acting on $W:=V \otimes_{E} V^{\prime}$ from the left and the other acting from the right. Therefore, we prefer to view the correspondence pairing an irreducible representation with its contragredient representation.

\subsection{Occurrence of representations of depth zero.}

Theorem. Let $\left(U(V), U\left(V^{\prime}\right)\right)$ be the reductive dual pair of unitary groups such that both $V, V^{\prime}$ are one-dimensional over an unramified quadratic extension of $F$. Let $\lambda_{F}$ denote the conductoral component of the fixed character $\psi$ of $F$. If $\lambda_{F}$ is even, then the trivial representation is the only depth zero representation that occurs in the theta correspondence. If $\lambda_{F}$ is odd, then every depth zero representation except the sgn character occurs in the theta correspondence.

Proof. Let $(\pi, \mathcal{V})$ be a depth zero irreducible representation of $U(V)$. Let $L$ be the lattice given in subsection 3.1. Because $L$ is the only good lattice in $V$, the restriction of the action of $\pi$ to $G_{L, 0^{+}}$must be trivial. Let $\bar{\pi}$ denote the action of $G_{L} / G_{L, 0^{+}}$on $\mathcal{V}$. We know that $\pi$ is uniquely determined by $\bar{\pi}$. By Proposition 2.8, we know that $\pi$ occurs in the theta correspondence for $\left(U(V), U\left(V^{\prime}\right)\right)$ if and only if $\bar{\pi}$ occurs in the theta correspondence for the dual pair $\left(G_{L} / G_{L, 0^{+}}, G_{L^{\prime}}^{\prime} / G_{L^{\prime}, 0^{+}}^{\prime}\right)$. Now if $\lambda_{F}$ is even, then $B\left(L, L^{\prime}\right):=L \otimes L^{\prime *}+L^{*} \otimes L^{\prime}$ is self-dual in $W$. Hence, $\operatorname{Sp}\left(B^{*} / B\right)$ is the trivial group $\operatorname{Sp}_{0}\left(\mathbf{f}_{F}\right)$. Therefore, the pair $\left(G_{L} / G_{L, 0^{+}}, G_{L^{\prime}}^{\prime} / G_{L^{\prime}, 0^{+}}^{\prime}\right)$ is $\left(\mathrm{U}_{1}\left(\mathbf{f}_{F}\right) \times \mathrm{U}_{0}\left(\mathbf{f}_{F}\right), \mathrm{U}_{0}\left(\mathbf{f}_{F}\right) \times \mathrm{U}_{1}\left(\mathbf{f}_{F}\right)\right)$ in $\mathrm{Sp}_{0}\left(\mathbf{f}_{F}\right)$. Hence, the only correspondence is the trivial representation to the trivial representation.

Next, if $\lambda_{F}$ is odd, then $B\left(L, L^{\prime}\right)$ is not self-dual. In fact, $B^{*} / B$ is two-dimensional over $\mathbf{f}_{F}$, and $\left(G_{L} / G_{L, 0^{+}}, G_{L^{\prime}}^{\prime} / G_{L^{\prime}, 0^{+}}^{\prime}\right)$ is the dual pair $\left(\mathrm{U}_{1}\left(\mathbf{f}_{F}\right) \times \mathrm{U}_{0}\left(\mathbf{f}_{F}\right), \mathrm{U}_{1}\left(\mathbf{f}_{F}\right) \times\right.$ $\left.\mathrm{U}_{0}\left(\mathbf{f}_{F}\right)\right)$ in $\operatorname{Sp}_{2}\left(\mathbf{f}_{F}\right)$. From the result in subsection 2.4 we know that the Weil representation of $\operatorname{Sp}_{2}\left(\mathbf{f}_{F}\right)$ is $q$-dimensional, and the unique character of order two of $\mathrm{U}_{1}\left(\mathbf{f}_{F}\right) \times \mathrm{U}_{0}\left(\mathbf{f}_{F}\right)$ is the only character that does not occur is the correspondence for $\left(G_{L} / G_{L, 0^{+}}, G_{L^{\prime}}^{\prime} / G_{L^{\prime}, 0^{+}}^{\prime}\right)$. 
3.4. Now we want to consider the correspondence of representations of positive depths. We need an easy lemma.

Lemma. Let $n_{0}, n_{0}^{\prime}$ be given as in subsection 3.1. Then $n_{0}+n_{0}^{\prime} \equiv \kappa+\kappa^{\prime}(\bmod 2)$.

Proof. From subsection 3.1 we know that $n_{0}=0$ if $\kappa$ is even, $n_{0}=1$ if $\kappa$ is odd. We also know that $n_{0}=0$ if $\kappa$ is even, $n_{0}=1$ if $\kappa$ is odd.

\subsection{Occurrence of representations of positive depth (I).}

Theorem. Keep the assumption in Theorem 3.3. Let $(\pi, \mathcal{V})$ be an irreducible admissible representation of $U(V)$ of positive depth $d$. If $d$ and $\lambda_{F}$ are of the same parity, then $\pi$ does not occur in the theta correspondence for the reductive dual pair $\left(U(V), U\left(V^{\prime}\right)\right)$.

Proof. Let $\mathcal{L}$ be defined as in subsection 3.1. Because $\mathcal{L}$ is the only regular small admissible lattice chain in $V$, the representation $\pi$ has a minimal $K$-type of the form $\left(G_{\mathcal{L}, d}, \zeta\right)$ for some nondegenerated character $\zeta$ of $G_{\mathcal{L}, d} / G_{\mathcal{L}, d^{+}}$. From Proposition 2.9 if $\pi$ occurs in the theta correspondence for the pair $\left(U(V), U\left(V^{\prime}\right)\right)$, then $V^{\prime}$ must have a regular small admissible lattice chain with numerical invariant $\left(1, n_{0}^{\prime}\right)$ such that $1+n_{0}+n_{0}^{\prime}+d$ is even. By Lemma $3.4 n_{0}+n_{0}^{\prime}$ and $\kappa+\kappa^{\prime}=\lambda_{F}$ are of the same parity. Therefore, $d$ and $\lambda_{F}$ can not be of the same parity. Hence, if $d$ and $\lambda_{F}$ are of the same parity, then $\pi$ can not occur in the theta correspondence for the pair $\left(U(V), U\left(V^{\prime}\right)\right)$.

3.6. Occurrence of representations of positive depth (II). Now we have the complement of Theorem 3.5.

Theorem. Keep the assumption in Theorem 3.3. Let $(\pi, \mathcal{V})$ be an irreducible representation of $U(V)$ of positive depth $d$. If $d$ and $\lambda_{F}$ are of the opposite parity, then $\pi$ occurs in the theta correspondence for the reductive dual pair $\left(U(V), U\left(V^{\prime}\right)\right)$.

Proof. Let $L, L^{\prime}$ be the unique good lattices in $V, V^{\prime}$, respectively, as in subsection 3.1. First we suppose that $\lambda_{F}$ is even. Then $B:=B\left(L, L^{\prime}\right)$ (see (2.8.1)) is a self-dual lattice in $W$. Let $\mathcal{S}(B)$ be the lattice model of the Weil representation with respect to $B$ and $\psi$. Let $k$ be a nonnegative integer, and let $w$ be a vector in $B \varpi_{F}^{-k-1}-B \varpi_{F}^{-k}$. Clearly, the space $\mathcal{S}(B)_{w}$ of functions in $\mathcal{S}(B)$ supported in $B+w$ is fixed by both $G_{\mathcal{L}, 2 k+2}$ and $G_{\mathcal{L}^{\prime}, 2 k+2}^{\prime}$. Therefore, $G_{\mathcal{L}, 2 k+1}$ (resp. $G_{\mathcal{L}^{\prime}, 2 k+1}^{\prime}$ ) acts on the space $\mathcal{S}(B)_{w}$ via the character $\mathfrak{M}(w)+\mathfrak{g}_{\mathcal{L},(-2 k-1)^{+}}^{*}\left(\right.$ resp. $\left.\mathfrak{M}^{\prime}(w)+\mathfrak{g}_{\mathcal{L}^{\prime},(-2 k-1)^{+}}\right)$. Note that it is a nondegenerate representation of $G_{\mathcal{L}, 2 k+1}$ i.e., it is a character of depth $2 k+1$. Now the dimension of the space $\sum_{w \in B \varpi_{F}^{-k-1}-B \varpi_{F}^{-k}} \mathcal{S}(B)_{w}$ is equal to $\left|\mathbf{f}_{E}\right|^{k+1}-\left|\mathbf{f}_{E}\right|^{k}=q^{2 k+2}-q^{2 k}$, where $q=\left|\mathbf{f}_{F}\right|$ denotes the cardinality of the finite field $\mathbf{f}_{F}$. The number of characters of $U(V)$ of depth $2 k+1$ is $(q+1) q^{2 k}(q-1)=$ $q^{2 k+2}-q^{2 k}$. But we know the multiplicity of any representation occurring in the local theta correspondence is exactly one. Therefore, every character of odd depth of $U(V)$ should occur in the theta correspondence.

Next we suppose that $\lambda_{F}$ is odd. Now $B\left(L, L^{\prime}\right)$ is not self-dual. Let $A_{0}$ be a selfdual lattice in $\mathcal{W}$ such that $B \subseteq A_{0} \subseteq B^{*}$. If $w \in B^{*} \varpi_{F}^{-k}-B^{*} \varpi_{F}^{-k+1}$ for some positive integer $k$, then $\mathcal{S}\left(A_{0}\right)_{w}$ is fixed by $G_{\mathcal{L}, 2 k+1}$ and acted by $G_{\mathcal{L}, 2 k}$ as a nondegenerate representation. Now the dimension of the space $\sum_{w \in B^{*} \varpi_{F}^{-k}-B^{*} \varpi_{F}^{-k+1}} \mathcal{S}\left(A_{0}\right)_{w}$ is equal to $q\left(\left|\mathbf{f}_{E}\right|^{k}-\left|\mathbf{f}_{E}\right|^{k-1}\right)=q^{2 k+1}-q^{2 k-1}$. The number of characters of $U(\mathcal{V})$ of depth $2 k$ is $(q+1) q^{2 k-1}(q-1)=q^{2 k+1}-q^{2 k-1}$. Therefore, every character of even depth of $U(V)$ occurs in the theta correspondence. 


\section{Theta correspondence for $\left(\mathrm{U}_{1}(F), \mathrm{U}_{1}(F)\right)$ II}

In this section, we assume that $E$ is a ramified quadratic extension of $F$ (i.e., $e=2)$ and both $V, V^{\prime}$ are one-dimensional. As usual, let $G:=U(V)$ and $G^{\prime}:=$ $U\left(V^{\prime}\right)$.

4.1. Lattice chains in $V^{\prime}$. As in subsection 3.1, we assume that $V$ is a Hermitian space with the form denoted by $\langle$,$\rangle , and we assume that V^{\prime}$ is a skew-Hermitian space with the form $\langle,\rangle^{\prime}$ defined by $\langle,\rangle^{\prime}:=\delta\langle$,$\rangle , where \delta$ is a number in $E^{\times}$such that $\tau(\delta)=-\delta$. For simplicity, we shall assume that $\operatorname{ord}(\delta)=\frac{1}{2}$. Now we have $2 \lambda_{F}-1=\kappa+\kappa^{\prime}$, because $E$ is ramified over $F$. Therefore, one of $\kappa$ and $\kappa^{\prime}$ is odd and the other is even.

Let $v_{0}$ be an element in $V$ such that $\operatorname{ord}\left(\left\langle v_{0}, v_{0}\right\rangle\right)=0$. Let $L$ be the lattice $v_{0} \mathfrak{p}_{E}^{\lceil\kappa / 2\rceil}$ in $V$. It is clear that $L$ is the unique good lattice in $V$. Moreover, $L$ is self-dual if $\kappa$ is even, and $L=L^{*} \varpi_{E}$ if $\kappa$ is odd. Now $G_{L} / G_{L, 0^{+}} \simeq \mathrm{O}_{1}\left(\mathbf{f}_{F}\right) \times \operatorname{Sp}_{0}\left(\mathbf{f}_{F}\right)$.

Define $L_{i}:=L \varpi_{E}^{i}$ for each $i \in \mathbb{Z}$. Then it is clear that the lattice chain $\mathcal{L}:=\left\{L_{i} \mid\right.$ $i \in \mathbb{Z}\}$ is the unique regular small admissible lattice chain in $V$. The numerical invariant $\left(n, n_{0}\right)$ of $\mathcal{L}$ is $(1,0)$ if $\kappa$ is even, and $\left(n, n_{0}\right)=(1,1)$ if $\kappa$ is odd. Therefore, we have

$$
G_{\mathcal{L}, d / 2}=\left\{g \in G \mid(g-1) . L \subseteq L \varpi_{E}^{d}\right\}
$$

for any positive integer $d$. Note that $G_{\mathcal{L}, d / 2} / G_{\mathcal{L},(d / 2)^{+}}$is isomorphic to $\mathbf{f}_{E}$ if $d$ is odd, and is the trivial group if $d$ is positive and even. Therefore, the possible depths of irreducible representations of $U(V)$ are zero or odd positive integers over 2 .

There is a unique good lattice $L^{\prime}$ in $V^{\prime}$. Moreover, $L^{\prime}$ is self-dual if $\kappa^{\prime}$ is odd and $L^{\prime}=L^{\prime *} \varpi_{E}$ if $\kappa^{\prime}$ is even. Let $\mathcal{L}^{\prime}:=\left\{L_{i}^{\prime} \mid i \in \mathbb{Z}\right\}$ be the unique regular small admissible lattice chain in $V^{\prime}$. The numerical invariant $\left(n^{\prime}, n_{0}^{\prime}\right)$ of $\mathcal{L}^{\prime}$ is $(1,1)$ if $\kappa^{\prime}$ is even, and $\left(n^{\prime}, n_{0}^{\prime}\right)=(1,0)$ if $\kappa^{\prime}$ is odd.

4.2. Occurrence of representations of depth zero. Now we consider the depth zero characters of $U(V)$. We know that $G_{L} / G_{L, 0^{+}} \simeq \mathrm{O}_{1}\left(\mathbf{f}_{F}\right) \times \operatorname{Sp}_{0}\left(\mathbf{f}_{F}\right)$. Therefore, $U(V)$ has exactly two characters of depth zero.

Theorem. Let $\left(U(V), U\left(V^{\prime}\right)\right)$ be the reductive dual pair of unitary groups such that both $V, V^{\prime}$ are one-dimensional over a ramified quadratic extension of $F$. The trivial character is the only depth zero representation of $U(V)$ that occurs in the theta correspondence for the dual pair $\left(U(V), U\left(V^{\prime}\right)\right)$.

Proof. Let $L$ (resp. $L^{\prime}$ ) be the unique good lattice in $V$ (resp. $V^{\prime}$ ). It is not difficult to check that the lattice $B:=B\left(L, L^{\prime}\right)$ is self-dual no matter where $\lambda_{F}$ is even or odd. Moreover, we know that $G_{L} / G_{L, 0^{+}} \simeq \mathrm{O}_{1}\left(\mathbf{f}_{F}\right) \times \operatorname{Sp}_{0}\left(\mathbf{f}_{F}\right)$ and $G_{L^{\prime}}^{\prime} / G_{L^{\prime}, 0^{+}}^{\prime} \simeq \operatorname{Sp}_{0}\left(\mathbf{f}_{F}\right) \times \mathrm{O}_{1}\left(\mathbf{f}_{F}\right)$. Hence, $\left(G_{L} / G_{L, 0^{+}}, G_{L^{\prime}}^{\prime} / G_{L^{\prime}, 0^{+}}^{\prime}\right)$ is the pair $\left(\mathrm{O}_{1}\left(\mathbf{f}_{F}\right) \times \operatorname{Sp}_{0}\left(\mathbf{f}_{F}\right), \operatorname{Sp}_{0}\left(\mathbf{f}_{F}\right) \times \mathrm{O}_{1}\left(\mathbf{f}_{F}\right)\right.$ in $\operatorname{Sp}_{0}\left(\mathbf{f}_{F}\right)$. Therefore, the only correspondence for $\left(G_{L} / G_{L, 0^{+}}, G_{L^{\prime}}^{\prime} / G_{L^{\prime}, 0^{+}}^{\prime}\right)$ is the trivial representation corresponding to the trivial representation. Therefore, by Proposition 2.8 the only depth zero representation of $U(V)$ that occurs in the theta correspondence for the pair $\left(U(V), U\left(V^{\prime}\right)\right)$ is the trivial representation. 


\subsection{Occurrence of representations of positive depths.}

Theorem. Keep the assumption in Theorem 3.3. Then exactly half of the characters of a given positive depth of $U(V)$ occurs in the theta correspondence for the reductive dual pair $\left(U(V), U\left(V^{\prime}\right)\right)$.

Proof. Let $L$ (resp. $L^{\prime}$ ) be the unique good lattice in $V$ (resp. $V^{\prime}$ ), respectively, as given in subsection 4.1. Then $B\left(L, L^{\prime}\right)$ is a self-dual lattice in $W$. As in the proof of Theorem 3.6, $\mathcal{S}(B)$ is the lattice model of the Weil representation. Now suppose that $k$ is a nonnegative integer and $w \in B \varpi_{E}^{-k-1}-B \varpi_{E}^{-k}$. Then the action on the space $\mathcal{S}(B)_{w}$ of $G_{\mathcal{L},(2 k+1) / 2}\left(\operatorname{resp} . G_{\mathcal{L}^{\prime},(2 k+1) / 2}^{\prime}\right)$ via $\mathfrak{M}(w)+\mathfrak{g}_{\mathcal{L},((-2 k-1) / 2)^{+}}^{*}$ (resp. $\mathfrak{M}^{\prime}(w)+\mathfrak{g}_{\mathcal{L}^{\prime},((-2 k-1) / 2)^{+}}^{\prime *}$ ) is a nondegenerate representation. The images of the maps

$$
\begin{aligned}
& B \varpi_{E}^{-k-1}-B \varpi_{E}^{-k} \rightarrow \mathfrak{g}_{\mathcal{L},(-2 k-1) / 2}^{*} / \mathfrak{g}_{\mathcal{L},((-2 k-1) / 2)^{+}}^{*} ; \\
& B \varpi_{E}^{-k-1}-B \varpi_{E}^{-k} \rightarrow \mathfrak{g}_{\mathcal{L}^{\prime},(-2 k-1) / 2}^{\prime *} / \mathfrak{g}_{\mathcal{L}^{\prime},((-2 k-1) / 2)^{+}}^{*}
\end{aligned}
$$

are only a subset of index two because $E$ is a ramified quadratic extension and $\mathfrak{M}, \mathfrak{M}^{\prime}$ are just the norm maps multiplied by $2 \delta$. Now the dimension of the space

$$
\sum_{w \in B \varpi_{E}^{-k-1}-B \varpi_{E}^{-k}} \mathcal{S}(B)_{w}
$$

is $q^{k+1}-q^{k}$, and the number of characters of $U(V)$ of depth $\frac{2 k+1}{2}$ is $2 q^{k}(q-1)=$ $2\left(q^{k+1}-q^{k}\right)$. Therefore, exactly half of the characters of $U(V)$ of depth $\frac{2 k+1}{2}$ occur in the theta correspondence for every nonnegative integer $k$.

\section{Theta CORRespondence For $\left(\mathrm{U}_{1}(F), \mathrm{U}_{1,1}(F)\right)$ I}

In this section we assume that $E$ is an unramified quadratic extension of $F, V$ is a one-dimensional Hermitian space and $V^{\prime}$ is a two-dimensional skew-Hermitian space with Witt index one.

5.1. Lattice chains in $V^{\prime}$. Let $G:=U(V), G^{\prime}:=U\left(V^{\prime}\right)$ and $\left(\omega_{\psi}, \mathcal{S}\right)$ be the Weil representation of $\widehat{\operatorname{Sp}(\mathcal{W})}$. Without loss of generality, we assume that $\kappa$ is 0 .

Let $v_{1}$ be an element in $V$ such that $\left\langle v_{1}, v_{1}\right\rangle=1$. Suppose that $\left\{v_{1}^{\prime}, v_{2}^{\prime}\right\}$ is a basis $V^{\prime}$ such that

$$
\left\langle v_{1}^{\prime}, v_{1}^{\prime}\right\rangle^{\prime}=\left\langle v_{2}^{\prime}, v_{2}^{\prime}\right\rangle^{\prime}=0, \quad\left\langle v_{1}^{\prime}, v_{2}^{\prime}\right\rangle^{\prime}=-\left\langle v_{2}^{\prime}, v_{1}^{\prime}\right\rangle^{\prime}=\varpi_{E}^{\kappa^{\prime}}
$$

where $\langle,\rangle^{\prime}$ denotes the skew-Hermitian form on $V^{\prime}$. Then the two lattices

$$
\begin{aligned}
M_{0}^{\prime} & :=v_{1}^{\prime} \mathcal{O}_{E}+v_{2}^{\prime} \mathcal{O}_{E} \\
M_{1}^{\prime} & :=v_{1}^{\prime} \mathfrak{p}_{E}+v_{2}^{\prime} \mathcal{O}_{E}
\end{aligned}
$$

are the only good lattices in $V^{\prime}$ up to equivalence. Let $L^{\prime}$ be a good lattice in $V^{\prime}$. It is easy to see that $G_{L^{\prime}}^{\prime} / G_{L^{\prime}, 0^{+}}^{\prime}$ is isomorphic to $\mathrm{U}_{2}\left(\mathbf{f}_{F}\right) \times \mathrm{U}_{0}\left(\mathbf{f}_{F}\right)$.

There are three regular small admissible lattice chains in $V^{\prime}$ which are generated by $\left\{M_{0}^{\prime}\right\},\left\{M_{1}^{\prime}\right\}$ and $\left\{M_{0}^{\prime}, M_{1}^{\prime}\right\}$, respectively. Their numerical invariants are $(1,0)$, $(1,1)$ and $(2,0)$, respectively. 
5.2. Occurrence. Now the dual pair $\left(U(V), U\left(V^{\prime}\right)\right)$ is in stable range, that is, the Witt index of $V^{\prime}$ is greater than or equal to the dimension of $V$. Thus all characters of $U(V)$ occur in the theta correspondence. Now $U(V)$ is compact. All its characters are supercuspidal. The trivial character of $U(V)$ occurs in the theta correspondence for the dual pair $\left(U(V), U\left(V^{\prime \prime}\right)\right)$, where $V^{\prime \prime}$ is the zero-dimensional " $\epsilon$-Hermitian space". Therefore, every character of $U(V)$ except the trivial one first occurs in the theta correspondence for the dual pair $\left(U(V), U\left(V^{\prime}\right)\right)$.

5.3. Correspondence of representations of depth zero. Let $(\pi, \mathcal{V})$ be a nontrivial irreducible representation of $U(V)$. Let $\left(\pi^{\prime}, \mathcal{V}^{\prime}\right)$ be the supercuspidal representation of $U\left(V^{\prime}\right)$ corresponding to $\pi$ in the theta correspondence for the pair $\left(U(V), U\left(V^{\prime}\right)\right)$. In this subsection we assume that $\pi$ is of depth zero. From subsection 4.1 we know that there is a unique good lattice $L$ in $V$. Because $\pi$ is of depth 0 , it is trivial on $G_{L, 0^{+}}$and can be regarded as a representation of $G_{L} / G_{L, 0^{+}} \simeq \mathrm{U}_{1}\left(\mathbf{f}_{F}\right) \times \mathrm{U}_{0}\left(\mathbf{f}_{F}\right)$. From the result in subsection 2.8 we know that there is a good lattice $L^{\prime}$ in $V^{\prime}$ such that $\pi \otimes$ triv (as a representation of $\left.G_{L} / G_{L, 0^{+}}\right)$first occurs in the theta correspondence for the finite reductive dual pair $\left(G_{L} / G_{L, 0^{+}}, G_{L^{\prime}}^{\prime} / G_{L^{\prime}, 0^{+}}^{\prime}\right)$. Hence, from subsection 2.4 we know that $\pi \otimes$ triv is paired with $\eta^{\prime} \otimes$ triv in the correspondence for the pair $\left(\mathrm{U}_{1}\left(\mathbf{f}_{F}\right) \times \mathrm{U}_{0}\left(\mathbf{f}_{F}\right), \mathrm{U}_{2}\left(\mathbf{f}_{F}\right) \times \mathrm{U}_{0}\left(\mathbf{f}_{F}\right)\right)$, where $\eta^{\prime}$ is a $(q-1)$-dimensional irreducible cuspidal representation of $\mathrm{U}_{2}\left(\mathbf{f}_{F}\right)$. Hence, we know that the representation $\pi^{\prime}$ has a minimal $K$-type $\left(G_{L^{\prime}}^{\prime}, \eta^{\prime} \otimes\right.$ triv $)$. Regard $\eta^{\prime} \otimes$ triv as a representation of $G_{L^{\prime}}^{\prime}$. The compactly induced representation $c$-Ind $G_{G^{\prime}}^{G^{\prime}}\left(\eta^{\prime} \otimes\right.$ triv) is irreducible and supercuspidal ( $c f$. [MP96]). Moreover, $c$-Ind $G_{G^{\prime}}^{G^{\prime}}\left(\eta^{\prime} \otimes\right.$ triv $)$ is the only irreducible representation of $G^{\prime}$ having minimal $K$ type $\left(G_{L^{\prime}}^{\prime}, \eta^{\prime}\right)$. Hence, $\pi^{\prime}$ is isomorphic to $c$ - $\operatorname{Ind}_{G_{L^{\prime}}^{\prime}}^{G^{\prime}}\left(\eta^{\prime} \otimes \operatorname{triv}\right)$.

5.4. Moment maps. Starting from this subsection to the end of this section, we are going to determine the correspondence for representations of positive depths. The final result is stated in subsection 5.9. We need a few lemmas first.

The Lie algebra $\mathfrak{g}^{\prime}$ of $U\left(V^{\prime}\right)$ can be identified with the space of two by two matrices of the form

$$
\left[\begin{array}{cc}
x & y \\
z & -\tau(x)
\end{array}\right]
$$

where $x \in E, y, z \in F$. We identify the Lie algebra $\mathfrak{g}$ of $U(V)$ with $\delta F$ as in subsection 4.1. Then it is easy to check that the moment maps $\mathfrak{M}$ and $\mathfrak{M}^{\prime}$ are given by

$$
\begin{aligned}
\mathfrak{M}\left(x v_{1} \otimes v_{1}^{\prime}+y v_{1} \otimes v_{2}^{\prime}\right) & =\varpi_{F}^{1-\lambda_{F}}(x \tau(y)-\tau(x) y), \\
\mathfrak{M}^{\prime}\left(x v_{1} \otimes v_{1}^{\prime}+y v_{1} \otimes v_{2}^{\prime}\right) & =-\varpi_{F}^{1-\lambda_{F}}\left[\begin{array}{cc}
-x \tau(y) & x \tau(x) \\
-y \tau(y) & \tau(x) y
\end{array}\right],
\end{aligned}
$$

where $v_{i}, v_{j}^{\prime}$ are given in subsection 5.1. Clearly the map $\mathfrak{M}$ is surjective.

5.5. Decomposition of $V^{\prime}$. Now we suppose that $\pi$ is an irreducible representation of $U(V)$ of positive depth. From subsection 4.1 we know that there is a unique regular small admissible lattice chain $\mathcal{L}=\left\{L_{i} \mid i \in \mathbb{Z}\right\}$ in $V$ and $\pi$ has a minimal $K$-type $\left(G_{\mathcal{L}, d / 1}, \zeta\right)$ for some positive integer $d$ and some $\zeta$. Let $\left(1, n_{0}\right)$ denote the numerical invariant of $\mathcal{L}$. From Proposition 2.9 we know that there are a regular small admissible lattice chain $\mathcal{L}^{\prime}=\left\{L_{i}^{\prime} \mid i \in \mathbb{Z}\right\}$ in $V^{\prime}$ with numerical invariant 
$\left(1, n_{0}^{\prime}\right)$ and an element $w_{0} \in B\left(\mathcal{L}, \mathcal{L}^{\prime}, \frac{d}{1}\right)$, such that $\left(G_{\mathcal{L}, d / 1}, \mathfrak{M}\left(w_{0}\right)+\mathfrak{g}_{\mathcal{L},-d^{+}}^{*}\right)$ is a minimal $K$-type of $\pi$ and $\left(G_{\mathcal{L}^{\prime}, d / 1}^{\prime}, \mathfrak{M}^{\prime}\left(w_{0}\right)+\mathfrak{g}_{\mathcal{L}^{\prime},-d^{+}}^{\prime *}\right)$ is a minimal $K$-type of $\pi^{\prime}$.

From (5.4.1) we know that $\mathfrak{M}^{\prime}\left(w_{0}\right)$ is a matrix of rank 1. Let $V_{1}^{\prime}$ (resp. $V_{2}^{\prime}$ ) be the one-dimensional subspace of $V^{\prime}$ spanned by the eigenvector corresponding to the nonzero (resp. zero) eigenvalue. Then $V^{\prime}$ is the orthogonal direct sum of $V_{1}^{\prime}$ and $V_{2}^{\prime}$.

Lemma. Let $L^{\prime}$ be the good lattice in $\mathcal{L}^{\prime}$. Then $L^{\prime}=\left(L^{\prime} \cap V_{1}^{\prime}\right) \oplus\left(L^{\prime} \cap V_{2}^{\prime}\right)$.

Proof. First suppose that $d$ is odd. Now $L=v_{1} \mathcal{O}_{E}, n=1, n_{0}=0$ and $n_{0}^{\prime}=0$. Hence, $L^{\prime}=v_{1}^{\prime} \mathcal{O}_{E}+v_{2}^{\prime} \mathcal{O}_{E}$. Moreover,

$$
B\left(\mathcal{L}, \mathcal{L}^{\prime}, \frac{d}{1}\right)=\mathfrak{p}_{E}^{-(d+1) / 2} v_{1} \otimes v_{1}^{\prime}+\mathfrak{p}_{E}^{-(d+1) / 2} v_{1} \otimes v_{2}^{\prime} .
$$

Let $w_{0}$ be the element in $B\left(\mathcal{L}, \mathcal{L}^{\prime}, \frac{d}{1}\right)$ such that $\mathfrak{M}\left(w_{0}\right)+\mathfrak{g}_{\mathcal{L},(-d / 1)^{+}}$presents a minimal $K$-type of $(\pi, \mathcal{V})$ as given before this lemma. Write

$$
w_{0}=x_{0} v_{1} \otimes v_{1}^{\prime}+y_{0} v_{1} \otimes v_{2}^{\prime}
$$

for some $x_{0}, y_{0} \in E$. Clearly, ord $\left(x_{0}\right) \geq-\frac{d+1}{2}$ and $\operatorname{ord}\left(y_{0}\right) \geq-\frac{d+1}{2}$. We claim that $\operatorname{ord}\left(x_{0}\right)=\operatorname{ord}\left(y_{0}\right)=-\frac{d+1}{2}$. If $\operatorname{ord}\left(x_{0}\right)>-\frac{d+1}{2}$, then $w_{0} \in \mathfrak{p}_{E}^{-(d-1) / 2} v_{1} \otimes$ $v_{1}^{\prime}+\mathfrak{p}_{E}^{-(d+1) / 2} v_{1} \otimes v_{2}^{\prime}$, which is $B\left(\mathcal{L}, \mathcal{L}^{\prime \prime}, \frac{d-1}{2}\right)$, where $\mathcal{L}^{\prime \prime}$ is the small admissible lattice chain with numerical invariant $(1,1)$ generated by the good lattice $L^{\prime \prime}=$ $\mathfrak{p}_{E} v_{1}^{\prime}+\mathcal{O}_{E} v_{2}^{\prime}$. Then $\mathfrak{M}\left(w_{0}\right)$ is in $\mathfrak{g}_{\mathcal{L},-(d-1) / 1}^{*}$. This means that the representation $\pi$ has nontrivial vectors fixed by $G_{\mathcal{L},((d-1) / 1)^{+}}$. Then the depth of $\pi$ is less than $d$. This contradicts the assumption that the depth of $\pi$ is $d$. Therefore, we have $\operatorname{ord}\left(x_{0}\right)=-\frac{d+1}{2}$. By the similar argument we can show that $\operatorname{ord}\left(y_{0}\right)=-\frac{d+1}{2}$. Now $L^{\prime}=v_{1}^{\prime} \mathcal{O}_{E}+v_{2}^{\prime} \mathcal{O}_{E}, V_{1}^{\prime}$ is spanned by the vector $v_{1}^{\prime} x_{0}+v_{2}^{\prime} y_{0}$ and $V_{2}^{\prime}$ is spanned by the vector $v_{1}^{\prime} \tau\left(x_{0}\right)+v_{2}^{\prime} \tau\left(y_{0}\right)$. Hence, $L^{\prime}=\left(L^{\prime} \cap V_{1}^{\prime}\right) \oplus\left(L^{\prime} \cap V_{2}^{\prime}\right)$.

Next suppose that $d$ is even. Now $n_{0}=0$ and $n_{0}^{\prime}=1, L=v_{1} \mathcal{O}_{E}$ and $L^{\prime}=$ $v_{1}^{\prime} \mathfrak{p}_{E}+v_{2}^{\prime} \mathcal{O}_{E}$. Hence,

$$
B\left(\mathcal{L}, \mathcal{L}^{\prime}, \frac{d}{1}\right)=\mathfrak{p}_{E}^{-d / 2} v_{1} \otimes v_{1}^{\prime}+\mathfrak{p}_{E}^{-(d+2) / 2} v_{1} \otimes v_{2}^{\prime} .
$$

Write $w_{0}=x_{0} v_{1} \otimes v_{1}^{\prime}+y_{0} v_{1} \otimes v_{2}^{\prime}$ for some $x_{0}, y_{0} \in E$. Clearly, ord $\left(x_{0}\right) \geq-\frac{d}{2}$ and $\operatorname{ord}\left(y_{0}\right) \geq-\frac{d+2}{2}$. We claim that $\operatorname{ord}\left(x_{0}\right)=-\frac{d}{2}$ and $\operatorname{ord}\left(y_{0}\right)=-\frac{d+2}{2}$. If $\operatorname{ord}\left(y_{0}\right)>$ $-\frac{d+2}{2}$, then $w_{0} \in \mathfrak{p}_{E}^{-d / 2} v_{1} \otimes v_{1}^{\prime}+\mathfrak{p}_{E}^{-d / 2} v_{1} \otimes v_{2}^{\prime}$, which is $B\left(\mathcal{L}, \mathcal{L}^{\prime \prime}, \frac{d-1}{2}\right)$, where $\mathcal{L}^{\prime \prime}$ is the small admissible lattice chain with numerical invariant $(1,0)$ generated by the good lattice $\mathcal{O}_{E} v_{1}^{\prime}+\mathcal{O}_{E} v_{2}^{\prime}$. This contradicts the assumption that the depth of $\pi$ is $d$. If $\operatorname{ord}\left(x_{0}\right)>-\frac{d}{2}$, then $w_{0} \in \mathfrak{p}_{E}^{-(d-2) / 2} v_{1} \otimes v_{1}^{\prime}+\mathfrak{p}_{E}^{-(d+2) / 2} v_{1} \otimes v_{2}^{\prime}$, which is $B\left(\mathcal{L}, \mathcal{L}^{\prime \prime}, \frac{d-1}{2}\right)$, where $\mathcal{L}^{\prime \prime}$ is the small admissible lattice chain with numerical invariant $(1,0)$ generated by the good lattice $\mathfrak{p}_{E} v_{1}^{\prime}+\mathfrak{p}_{E}^{-1} v_{2}^{\prime}$. Clearly the good lattice $\mathfrak{p}_{E} v_{1}^{\prime}+\mathfrak{p}_{E}^{-1} v_{2}^{\prime}$ is equivalent to $\mathcal{O}_{E} v_{1}^{\prime}+\mathcal{O}_{E} v_{2}^{\prime}$. Hence, this contradicts the assumption that the depth of $\pi$ is $d$ again. Therefore, we have ord $\left(x_{0}\right)=-\frac{d}{2}$ and $\operatorname{ord}\left(y_{0}\right)=-\frac{d+2}{2}$. Now $L^{\prime}=v_{1}^{\prime} \mathfrak{p}_{E}+v_{2}^{\prime} \mathcal{O}_{E}, V_{1}^{\prime}$ is spanned by the vector $v_{1}^{\prime} x_{0}+v_{2}^{\prime} y_{0}$ and $V_{2}^{\prime}$ is spanned by the vector $v_{1}^{\prime} \tau\left(x_{0}\right)+v_{2}^{\prime} \tau\left(y_{0}\right)$. Hence, $L^{\prime}=\left(L^{\prime} \cap V_{1}^{\prime}\right) \oplus\left(L^{\prime} \cap V_{2}^{\prime}\right)$, again.

Let $\langle,\rangle_{i}^{\prime}$ denote the restrictions of $\langle,\rangle^{\prime}$ to $V_{i}^{\prime} \times V_{i}^{\prime}$ for $i=1,2$. It is easy to see that both the forms $\langle,\rangle_{1}^{\prime},\langle,\rangle_{2}^{\prime}$ are nondegenerate. We can also check that the centralizer of $\mathfrak{M}^{\prime}\left(w_{0}\right)$ in $U\left(V^{\prime}\right)$ is $U\left(V_{1}^{\prime}\right) \times U\left(V_{2}^{\prime}\right)$. 
5.6. Decomposition of the Weil representation. Define $F$ spaces:

$$
W_{1}:=V \otimes V_{1}^{\prime}, \quad W_{2}:=V \otimes V_{2}^{\prime} .
$$

Then $W$ can be identified with the direct sum $W_{1} \oplus W_{2}$, and each $W_{i}$ is regarded as a subspace of $W$. It is not difficult to check that $w_{0} \in W_{1} \subset W$. Define

$$
\begin{aligned}
& \langle,\rangle_{1}:=\operatorname{Tr}_{E / F}\left(\langle,\rangle \otimes \tau\left(\langle,\rangle_{1}^{\prime}\right)\right), \\
& \langle,\rangle_{2}:=\operatorname{Tr}_{E / F}\left(\langle,\rangle \otimes \tau\left(\langle,\rangle_{2}^{\prime}\right)\right) .
\end{aligned}
$$

It is clear that $\left\langle\langle,\rangle_{1}\right.$ and $\langle,,\rangle_{2}$ are skew-symmetric bilinear forms on $W_{1}$ and $W_{2}$, respectively. It is also clear that $\left\langle\langle,\rangle_{i}\right.$ is the just the restriction of $\langle\langle\rangle$,$\rangle to W_{i} \times W_{i}$. So we know that $\left(U(V), U\left(V_{1}^{\prime}\right)\right)$ and $\left(U(V), U\left(V_{2}^{\prime}\right)\right)$ form reductive dual pairs in $\operatorname{Sp}\left(W_{1}\right)$ and $\operatorname{Sp}\left(W_{2}\right)$, respectively. Define

$$
B:=L_{0} \otimes L_{0}^{\prime *} \cap L_{0}^{*} \otimes L_{0}^{\prime} .
$$

We know that $B$ is a good lattice in $W$. Now it is easy to see that $B=L \otimes L^{\prime}$ because $L$ is self-dual (recall that we assume $\kappa=0$ in this section). Hence, we have

$$
B=\left(B \cap W_{1}\right) \oplus\left(B \cap W_{2}\right)
$$

from Lemma 5.5. Denote $B \cap W_{i}$ by $B_{i}$ for $i=1,2$. It is clear that $B_{i}$ is a good lattice in $W_{i}$. Let $B_{i}^{*}$ denote the dual lattice in $W_{i}$, and let $\mathbf{b}_{i}^{*}$ be the quotient $B_{i}^{*} / B_{i}$. From (5.6.3) we know that $\mathbf{b}^{*}=\mathbf{b}_{1}^{*} \oplus \mathbf{b}_{2}^{*}$, where $\mathbf{b}^{*}=B^{*} / B$. Regard $\operatorname{Sp}\left(\mathbf{b}_{1}^{*}\right) \times \operatorname{Sp}\left(\mathbf{b}_{2}^{*}\right)$ as a subgroup of $\operatorname{Sp}\left(\mathbf{b}^{*}\right)$. Then we know that $S \simeq S_{1} \otimes_{\mathbb{C}} S_{2}$ as a representation of $\operatorname{Sp}\left(\mathbf{b}_{1}^{*}\right) \times \operatorname{Sp}\left(\mathbf{b}_{2}^{*}\right)$, where $\left(\bar{\omega}_{i, \bar{\psi}}, S_{i}\right)$ is the Weil representation of the finite symplectic group $\operatorname{Sp}\left(\mathbf{b}_{i}^{*}\right)$. Let $\widetilde{\rho}_{i, \psi}$ denote the action of $\mathrm{H}\left(B_{i}^{*}\right)$ on $S_{i}$ (cf. subsection 2.6). It is clear that $\widetilde{\rho}_{\psi}\left(b_{1}+b_{2}\right)=\widetilde{\rho}_{1, \psi}\left(b_{1}\right) \otimes \widetilde{\rho}_{2, \psi}\left(b_{2}\right)$ for $b_{i} \in B_{i}^{*}$ when $S$ is identified with $S_{1} \otimes_{\mathbb{C}} S_{2}$.

Let $\widetilde{\operatorname{Sp}\left(W_{1}\right)}$ (resp. $\left.\widetilde{\operatorname{Sp}\left(W_{2}\right)}\right)$ be the metaplectic cover of $\operatorname{Sp}\left(W_{1}\right)$ (resp. $\operatorname{Sp}\left(W_{2}\right)$ ). Regard $\left(\omega_{\psi}, \mathcal{S}\right)$ as a representation of $\widetilde{\operatorname{Sp}\left(W_{1}\right)} \times \widetilde{\operatorname{Sp}\left(W_{2}\right)}$ via the composition of the homomorphism

$$
\widetilde{\operatorname{Sp}\left(W_{1}\right)} \times \widetilde{\operatorname{Sp}\left(W_{2}\right)} \rightarrow \operatorname{Sp}\left(W_{1} \widetilde{T} \times \operatorname{Sp}\left(W_{2}\right)\right.
$$

and the inclusion

$$
\operatorname{Sp}\left(W_{1} \widetilde{\widetilde{S}} \mathrm{p}\left(W_{2}\right) \hookrightarrow \widetilde{\operatorname{Sp}(W)} .\right.
$$

Let $\mathcal{S}\left(B_{1}\right)$ (resp. $\mathcal{S}\left(B_{2}\right)$ ) be the generalized lattice model of the Weil representation of $\operatorname{Sp(W_{1})}$ (resp. $\operatorname{Sp}\left(W_{2}\right)$ ) with respect to the character $\psi$. Recall that $\mathcal{S}\left(B_{i}\right)$ is the space of locally constant, compactly supported maps $f: W_{i} \rightarrow S_{i}$ such that

$$
f(b+x)=\psi\left(\frac{1}{2}\left\langle\langle x, b\rangle_{i}\right) \tilde{\rho}_{i, \psi}(b) . f(x),\right.
$$

for any $b \in B_{i}^{*}$, and $x \in W_{i}$. Define a map $\Xi: \mathcal{S}\left(B_{1}\right) \otimes_{\mathbb{C}} \mathcal{S}\left(B_{2}\right) \rightarrow \mathcal{S}(B)$ by

$$
\Xi\left(f_{1} \otimes f_{2}\right)\left(x_{1}+x_{2}\right):=f_{1}\left(x_{1}\right) \otimes f_{2}\left(x_{2}\right)
$$

for $x_{i} \in W_{i}$. We identify $S$ with $S_{1} \otimes_{\mathbb{C}} S_{2}$ in (5.6.4).

Lemma. The map $\Xi$ gives an isomorphism between $\mathcal{S}\left(B_{1}\right) \otimes_{\mathbb{C}} \mathcal{S}\left(B_{2}\right)$ and $\mathcal{S}(B)$ as a representation of $\widetilde{\operatorname{Sp}\left(W_{1}\right)} \times \widetilde{\operatorname{Sp}\left(W_{2}\right)}$.

Proof. First we check that $f:=\Xi\left(f_{1} \otimes f_{2}\right)$ is really in $\mathcal{S}(B)$. Recall that $W=$ $W_{1} \oplus W_{2}$ and $B=B_{1} \oplus B_{2}$. Suppose that $x$ is a vector in $W$ and $b$ is a an element 
in $B$. Then we can write $x=x_{1}+x_{2}$ for $x_{i} \in W_{i}$ and $b=b_{1}+b_{2}$ for $b_{i} \in B_{i}$. Therefore,

$$
\begin{aligned}
f(b+x) & =f\left(b_{1}+x_{1}+b_{2}+x_{2}\right) \\
& =f_{1}\left(b_{1}+x_{1}\right) \otimes f_{2}\left(b_{2}+x_{2}\right) \\
& \left.=\psi\left(\left\langle x_{1}, b_{1}\right\rangle\right\rangle_{1}\right) \widetilde{\rho}_{1, \psi}\left(b_{1}\right) \cdot f_{1}\left(x_{1}\right) \otimes \psi\left(\left\langle x_{2}, b_{2}\right\rangle_{2}\right) \widetilde{\rho}_{2, \psi}\left(b_{2}\right) \cdot f_{2}\left(x_{2}\right) \\
& =\psi\left(\left\langle\left(x_{1}, b_{1}\right\rangle\right\rangle_{1}+\left\langle\left\langle x_{2}, b_{2}\right\rangle_{2}\right) \widetilde{\rho}_{1, \psi}\left(b_{1}\right) \cdot f_{1}\left(x_{1}\right) \otimes \widetilde{\rho}_{2, \psi}\left(b_{2}\right) \cdot f_{2}\left(x_{2}\right)\right. \\
& \left.=\psi\left(\left\langle x_{1}+x_{2}, b_{1}+b_{2}\right\rangle\right)\right) \widetilde{\rho}_{\psi}\left(b_{1}+b_{2}\right) \cdot f\left(x_{1}+x_{2}\right) \\
& =\psi\left(\langle\langle x, b\rangle) \widetilde{\rho}_{\psi}(b) \cdot f(x) .\right.
\end{aligned}
$$

Hence, $\Xi\left(f_{1} \otimes f_{2}\right)$ is in $\mathcal{S}(B)$.

Next we want to prove that the map $\Xi$ intertwines the actions of $\widetilde{\operatorname{sp}\left(W_{1}\right)} \times$ $\widetilde{\operatorname{Sp}\left(W_{2}\right)}$. Let $f_{i} \in \mathcal{S}\left(B_{i}\right), g=\left(g_{1}, g_{2}\right) \in \operatorname{Sp}\left(W_{1}\right) \times \operatorname{Sp}\left(W_{2}\right)$ and $x \in W$. Then

$$
\begin{aligned}
\left(\left(M_{B}[g] \circ \Xi\right) \cdot f_{1} \otimes f_{2}\right)(x) & =\left(M_{B}[g] . f\right)(x) \\
& =\int_{B} \psi\left(\frac{1}{2}\langle\langle b, w\rangle)\right) \widetilde{\rho}_{\psi}\left(b^{-1}\right) \cdot f\left(g^{-1} \cdot(b+x)\right) d b .
\end{aligned}
$$

Because $B=B_{1} \oplus B_{2}$, any $b \in B$ can be written uniquely as $b=b_{1}+b_{2}$ for $b_{i} \in B_{i}$. Also $x$ can be written uniquely as $x_{1}+x_{2}$ for $x_{i} \in W_{i}$. Let $d b$ (resp. $d b_{1}, d b_{2}$ ) be the Haar measure of $B$ (resp. $B_{1}, B_{2}$ ) such that the volume of $B$ is equal to the product of volumes of $B_{1}$ and $B_{2}$. Hence,

$$
\begin{aligned}
&\left(\left(M_{B}[g] \circ \Xi\right) \cdot f_{1} \otimes f_{2}\right)(x) \\
&= \int_{B_{2}}\left(\int_{B_{1}} \psi\left(\frac{1}{2}\left\langle b_{1}+b_{2}, x_{1}+x_{2}\right\rangle\right)\right. \\
&\left.\widetilde{\rho}_{\psi}\left(\left(b_{1}+b_{2}\right)^{-1}\right) \cdot f\left(\left(g_{1}, g_{2}\right)^{-1} \cdot\left(b_{1}+x_{1}+b_{2}+x_{2}\right)\right) d b_{1}\right) d b_{2} \\
&=\left(\int_{B_{1}} \psi\left(\frac{1}{2}\left\langle b_{1}, x_{1}\right\rangle_{1}\right) \rho_{\psi}\left(b_{1}^{-1}\right) \cdot f\left(g_{1}^{-1} \cdot\left(b_{1}+x_{1}\right)\right) d b_{1}\right) \otimes \\
&=\left(\int_{B_{2}} \psi\left(\frac{1}{2}\left\langle b_{2}, x_{2}\right\rangle_{2}\right) \rho_{\psi}\left(b_{2}^{-1}\right) \cdot f\left(g_{2}^{-1} \cdot\left(b_{2}+x_{2}\right)\right) d b_{2}\right) \\
&=\left(M_{B_{1}}\left[g_{1}\right] \cdot f_{1}\right)\left(x_{1}\right) \otimes\left(M_{B_{2}}\left[g_{2}\right] \cdot f_{2}\right)\left(x_{2}\right) \\
&=\left.\left(\Xi \circ\left(M_{B_{1}}\left[g_{1}\right] \cdot f_{1}\right] \otimes M_{B_{2}}\left[g_{2}\right] \cdot f_{2}\right)\right)(x) \\
&\left.\left(\Xi M_{B_{2}}\left[g_{2}\right]\right) \cdot f_{1} \otimes f_{2}\right)(x) .
\end{aligned}
$$

Hence, $\Xi$ intertwines the actions $M_{B}\left[g_{1}, g_{2}\right]$ and $M_{B_{1}}\left[g_{1}\right] \otimes M_{B_{2}}\left[g_{2}\right]$ for any $g_{1} \in$ $\operatorname{Sp}\left(W_{1}\right)$ and $g_{2} \in \operatorname{Sp}\left(W_{2}\right)$. Clearly $\Xi$ is an isomorphism of vector spaces.

\section{7 .}

Lemma. $U\left(V_{1}^{\prime}\right) \times U\left(V_{2}^{\prime}\right)$ is a subgroup of $G_{L^{\prime}}^{\prime}$.

Proof. Let $L_{i}^{\prime}:=L^{\prime} \cap V_{i}^{\prime}$. We have $L^{\prime}=L_{1}^{\prime} \oplus L_{2}^{\prime}$ from Lemma 5.5. Moreover, $L_{i}^{\prime}$ is the unique good lattice in $V_{i}^{\prime}$. Because $V_{i}^{\prime}$ is anisotropic, the group $U\left(V_{i}^{\prime}\right)$ stabilizes the unique good lattice $L_{i}^{\prime}$. Hence, the group $U\left(V_{1}^{\prime}\right) \times U\left(V_{2}^{\prime}\right)$ stabilizes both $L_{1}^{\prime}$ and $L_{2}^{\prime}$. Therefore, $U\left(V_{1}^{\prime}\right) \times U\left(V_{2}^{\prime}\right)$ stabilizes $L^{\prime}$. Thus $U\left(V_{1}^{\prime}\right) \times U\left(V_{2}^{\prime}\right)$ is a subgroup of $G_{L^{\prime}}^{\prime}$

From this lemma we know that the splitting $U\left(V_{i}^{\prime}\right) \rightarrow \widetilde{U\left(V_{i}^{\prime}\right)}$ in $\widetilde{\operatorname{Sp}\left(W_{i}\right)}$ is compatible with the splitting $U\left(V^{\prime}\right) \rightarrow \widetilde{U\left(V^{\prime}\right)}$ in $\widetilde{\operatorname{Sp}(W)}$.

5.8. Decomposition of the local theta correspondence. In this subsection we want to describe $\pi^{\prime}$ in terms of data from $\pi$ when the depth of $\pi$ is positive. Recall that there is a surjective map

$$
\mathcal{S}(B) \rightarrow \mathcal{V} \otimes_{\mathbb{C}} \mathcal{V}^{\prime}
$$


From previous discussion we know that there is an element $w_{0} \in W$ such that $\mathcal{S}(B)_{w_{0}}$ has nontrivial image under the projection $\Pi: \mathcal{S}(B) \rightarrow \mathcal{V} \otimes_{\mathbb{C}} \mathcal{V}^{\prime}$. Let $f$ be an element in $\mathcal{S}(B)_{w_{0}}$ such that $\Pi(f)$ is nontrivial. The space spanned by the function $f_{\pi}$ defined by

$$
f_{\pi}:=\sum_{g \in G / G_{\mathcal{L},(d / 1)+}} \pi(g)^{-1} \omega_{\psi}(g) \cdot f
$$

is acted by $U(V)$, and the action is $\pi$ ( $c f$. Yan98).

The isomorphism $\mathcal{S}\left(B_{1}\right) \otimes_{\mathbb{C}} \mathcal{S}\left(B_{2}\right) \rightarrow \mathcal{S}(B)$ given in Lemma 5.6 maps the subspace $\mathcal{S}\left(B_{1}\right)_{w_{0}} \otimes_{\mathbb{C}} \mathcal{S}\left(B_{2}\right)_{0}$ onto $\mathcal{S}(B)_{w_{0}}$, where 0 denotes the zero vector in $W_{2}$ and $w_{0}$ is regarded as an element in both $W_{1}$ and $W$. Hence, $\mathcal{S}\left(B_{1}\right)_{w_{0}} \otimes_{\mathbb{C}} \mathcal{S}\left(B_{2}\right)_{0}$ has nontrivial image under the map

$$
\mathcal{S}\left(B_{1}\right) \otimes_{\mathbb{C}} \mathcal{S}\left(B_{2}\right) \rightarrow \mathcal{V} \otimes_{\mathbb{C}} \mathcal{V}^{\prime}
$$

Therefore, $(\pi, \mathcal{V})$, as a representation of $U(V) \times U(V)$, can be decomposed as the tensor product $\left(\pi_{1} \otimes \pi_{2}, \mathcal{V}_{1} \otimes \mathcal{V}_{2}\right)$, and $\left(\pi^{\prime}, \mathcal{V}^{\prime}\right)$, as a representation of $U\left(V_{1}^{\prime}\right) \times$ $U\left(V_{2}^{\prime}\right)$, has a subrepresentation $\left(\pi_{1}^{\prime} \otimes \pi_{2}^{\prime}, \mathcal{V}_{1}^{\prime} \otimes_{\mathbb{C}} \mathcal{V}_{2}^{\prime}\right)$ such that $\mathcal{S}\left(B_{1}\right)_{w_{0}} \otimes_{\mathbb{C}} \mathcal{S}\left(B_{2}\right)_{0}$ has nontrivial image under the $(U(V) \times U(V)) \times\left(U\left(V_{1}^{\prime}\right) \times U\left(V_{2}^{\prime}\right)\right)$ map

$$
\mathcal{S}\left(B_{1}\right) \otimes_{\mathbb{C}} \mathcal{S}\left(B_{2}\right) \rightarrow\left(\mathcal{V}_{1} \otimes_{\mathbb{C}} \mathcal{V}_{1}^{\prime}\right) \otimes_{\mathbb{C}}\left(\mathcal{V}_{2} \otimes_{\mathbb{C}} \mathcal{V}_{2}^{\prime}\right)
$$

Therefore, the representations $\pi_{1}, \pi_{1}^{\prime}$ are paired in the theta correspondence for the dual pair $\left(U(V), U\left(V_{1}^{\prime}\right)\right)$ in $\operatorname{Sp}\left(W_{1}\right)$ such that the subspace $\mathcal{S}\left(B_{1}\right)_{w_{0}}$ has nontrivial image, and $\pi_{2}, \pi_{2}^{\prime}$ are paired in the theta correspondence for the dual pair $\left(U(V), U\left(V_{2}^{\prime}\right)\right)$ in $\operatorname{Sp}\left(W_{2}\right)$ such that the subspace $\mathcal{S}\left(B_{2}\right)_{0}$ has nontrivial image. Hence, $\pi_{1}$ is isomorphic to $\pi$, and $\pi_{1}^{\prime}$ is isomorphic to the contragredient representation $\tilde{\pi}$ of $\pi$ if we identify $U\left(V_{1}^{\prime}\right)$ with $U(V)$. Moreover, it is easy to see that both groups $U(V)$ and $U\left(V_{2}^{\prime}\right)$ acts trivially on $\mathcal{S}\left(B_{2}\right)_{0}$. Hence, both $\pi_{2}, \pi_{2}^{\prime}$ are the trivial representations.

5.9. Correspondence of representations of positive depths. Let $G^{\prime}=U\left(V^{\prime}\right)$ and $\mathfrak{g}^{\prime}$ be the Lie algebra of $G^{\prime}$. Let $H:=U\left(V_{1}^{\prime}\right) \times U\left(V_{2}^{\prime}\right)$ and $\mathfrak{h}$ be the Lie algebra of $H$. Now we recall a result from Ad198. Let $\mathfrak{h}^{\perp}$ denote the perpendicular of $\mathfrak{h}$ in $\mathfrak{g}^{\prime}$. Let

$$
\begin{aligned}
\mathfrak{J}_{+} & :=\left(\mathfrak{h} \cap \mathfrak{g}_{\mathcal{L}, d}^{\prime}\right) \oplus\left(\mathfrak{h}^{\perp} \cap \mathfrak{g}_{\mathcal{L},(d / 2)+}^{\prime}\right), \\
\mathfrak{J} & :=\left(\mathfrak{h} \cap \mathfrak{g}_{\mathcal{L}, d}^{\prime}\right) \oplus\left(\mathfrak{h}^{\perp} \cap \mathfrak{g}_{\mathcal{L}, d / 2}^{\prime}\right) .
\end{aligned}
$$

For $X \in \mathfrak{g}^{\prime}$ such that $1-X$ is invertible we define a Cayley transform

$$
\Psi(X):=(1+X)(1-X)^{-1},
$$

which is an element in $U\left(V^{\prime}\right)$. It is easy to see that $1-X$ is invertible for any element $X$ in $\mathfrak{J}$. Define $J_{+}:=\Psi\left(\mathfrak{J}_{+}\right)$and $J:=\Psi(\mathfrak{J})$. It is known that $H$ normalizes $J$ and $J_{+}$. It is known from Adl98 that for an irreducible representation $\sigma$ of $H$ we can construct an irreducible admissible representation $\phi_{\sigma}$ of $H J$ with dimension equal to

$$
\operatorname{dim}(\sigma) \sqrt{\#\left(\left(\mathfrak{h}^{\perp} \cap \mathfrak{g}_{\mathcal{L}, d / 2}\right) /\left(\mathfrak{h}^{\perp} \cap \mathfrak{g}_{\mathcal{L},(d / 2)^{+}}\right)\right)} .
$$

It is also known from Ad198, that the compactly induced representation $c$-Ind $\operatorname{InJ}_{J}^{G^{\prime}} \phi_{\sigma}$ is an irreducible supercuspidal representation of $G^{\prime}$. Now we denote $\phi_{\sigma}$ by $\phi_{\tilde{\pi}}$ if $\sigma=\tilde{\pi} \otimes$ triv, where $\tilde{\pi}$ is the contragredient representation of a character $\pi$ of $U\left(V_{1}^{\prime}\right)$ and triv is the trivial representation of $U\left(V_{2}^{\prime}\right)$. 
Theorem. Let $\left(U(V), U\left(V^{\prime}\right)\right)$ be the reductive dual pair of unitary groups such that $V$ (resp. $\left.V^{\prime}\right)$ is one-dimensional (resp. two-dimensional with Witt index one) over an unramified quadratic extension of $F$. The theta correspondence for the dual pair $\left(U(V), U\left(V^{\prime}\right)\right)$ of irreducible representations of positive depths is the following:

$$
\pi \longleftrightarrow c-\operatorname{Ind}_{H}^{G^{\prime}} \phi_{\tilde{\pi}} .
$$

Proof. Suppose that a character $\pi$ of $U(V)$ of positive depth is paired with the irreducible supercuspidal representation $\pi^{\prime}$ of $U\left(V^{\prime}\right)$. From the discussion in subsections 5.5-5.8 we know that there is a decomposition $V^{\prime}=V_{1}^{\prime} \oplus V_{2}^{\prime}$ such that the restriction of $\pi^{\prime}$ to $H:=U\left(V_{1}^{\prime}\right) \times U\left(V_{2}^{\prime}\right)$ contains the character $\tilde{\pi} \otimes$ triv, where $\tilde{\pi}$ is the contragredient representation of $\pi$. By Proposition 2.6.3 of [Adl98] we know that the restriction of $\pi^{\prime}$ to $H J$ contains $\phi_{\tilde{\pi}}$. By the result in Ad198 again we know that there is only one irreducible (supercuspidal) representation of $U\left(V^{\prime}\right)$ whose restriction to $H J$ contains $\phi_{\tilde{\pi}}$. Hence, $\pi^{\prime}$ is isomorphic to $c-\operatorname{Ind}_{H J}^{G^{\prime}} \phi_{\tilde{\pi}}$.

\section{Theta CORRESPondence For $\left(\mathrm{U}_{1}(F), \mathrm{U}_{1,1}(F)\right)$ II}

In this section, we assume that $E$ is a ramified quadratic extension of $F, V$ is one-dimensional and $V^{\prime}$ is two-dimensional with Witt index one.

6.1. Lattice chains in $V^{\prime}$. As in subsection 4.1, there is a unique good $L$ lattice in $V$ and $G_{L} / G_{L, 0^{+}}$is isomorphic to $\mathrm{O}_{1}\left(\mathbf{f}_{F}\right) \times \operatorname{Sp}_{0}\left(\mathbf{f}_{F}\right)$. Similar to the situation in subsection 5.1 there are two good lattices in $V^{\prime}$, and $G_{L^{\prime}}^{\prime} / G_{L^{\prime}, 0^{+}}^{\prime}$ is either isomorphic to $\mathrm{Sp}_{2}\left(\mathbf{f}_{F}\right) \times \mathrm{O}_{0}\left(\mathbf{f}_{F}\right)$ or $\mathrm{Sp}_{0}\left(\mathbf{f}_{F}\right) \times \mathrm{O}_{1,1}\left(\mathbf{f}_{F}\right)$.

Let $M_{1}^{\prime} \subseteq M_{0}^{\prime}$ be the two good lattice in $V^{\prime}$. There are three regular small admissible lattice chains in $V^{\prime}$, which are generated by $\left\{M_{0}^{\prime}\right\},\left\{M_{1}^{\prime}\right\}$ and $\left\{M_{0}^{\prime}, M_{1}^{\prime}\right\}$, respectively. Their numerical invariants are $(1,0),(1,1)$ and $(2,0)$, respectively.

6.2. Occurrence. All characters of $U(V)$ occur in the theta correspondence because the dual pair is in stable range. Suppose $\pi$ is a nontrivial character of $U(V)$. Then $\pi$ first occurs in the theta correspondence for the dual pair $\left(U(V), U\left(V^{\prime}\right)\right)$. So $\pi$ is paired with an irreducible supercuspidal representation $\pi^{\prime}$ of $U\left(V^{\prime}\right)$ by the induction principle.

6.3. Correspondence of representations of depth zero. Suppose that $\pi$ is a nontrivial character of $U(V)$ of depth zero. As in subsection $4.1 \pi$ can be regarded as a nontrivial representation of $\mathrm{O}_{1}\left(\mathbf{f}_{F}\right) \times \operatorname{Sp}_{0}\left(\mathbf{f}_{F}\right)$. Hence, $\pi$ is isomorphic to $\operatorname{sgn} \otimes$ triv when regarded as a representation of $\mathrm{O}_{1}\left(\mathbf{f}_{F}\right) \times \mathrm{Sp}_{0}\left(\mathbf{f}_{F}\right)$. We know that $\operatorname{sgn} \otimes$ triv first occurs in the correspondence for the pair $\left(\mathrm{O}_{1}\left(\mathbf{f}_{F}\right) \times \operatorname{Sp}_{0}\left(\mathbf{f}_{F}\right), \operatorname{Sp}_{2}\left(\mathbf{f}_{F}\right) \times \mathrm{O}_{0}\left(\mathbf{f}_{F}\right)\right)$ and is paired with $\eta^{\prime} \otimes$ triv, where $\eta^{\prime}$ is a $\frac{q-1}{2}$-dimensional irreducible cuspidal representation of $\operatorname{Sp}_{2}\left(\mathbf{f}_{F}\right)$. Let $L^{\prime}$ be the good lattice in $V^{\prime}$ such that $G_{L^{\prime}}^{\prime} / G_{L, 0^{+}}^{\prime}$ is isomorphic to $\mathrm{SL}_{2}\left(\mathbf{f}_{F}\right) \times \mathrm{O}_{0}\left(\mathbf{f}_{F}\right)$. Then we know that $\pi^{\prime}$ is isomorphic to the induced representation $c$-Ind $\operatorname{IG}_{L^{\prime}}^{G^{\prime}}\left(\eta^{\prime} \otimes\right.$ triv $)$.

6.4. Correspondence of representations of positive depths. All the results in subsections 5.4 to 5.8 work when $E$ is a ramified extension. Therefore, the explicit correspondence of representations of positive depths is the same as the case for unramified $E$ in subsection 5.9. 


\section{ThetA CORRESPONDENCE FOR $\left(\mathrm{U}_{1}(F), \mathrm{U}_{2}(F)\right)$ I}

In this section, we assume that $E$ is an unramified quadratic extension of $F, V$ is one-dimensional and $V^{\prime}$ is two-dimensional anisotropic.

7.1. Lattice chains in $V^{\prime}$. In this subsection we assume that $E=F(\sqrt{\alpha})$, where $\alpha \in \mathcal{O}_{F}^{*}$ is not a square. Let $\delta:=\sqrt{\alpha}$. Then $\tau(\delta)=-\delta$. We can find a basis $\left\{v_{1}^{\prime}, v_{2}^{\prime}\right\}$ of $V^{\prime}$ such that the form $\langle,\rangle^{\prime}$ on $V^{\prime}$ is given by

$$
\left\langle v_{1}^{\prime} x_{1}+v_{2}^{\prime} y_{1}, v_{1}^{\prime} x_{2}+v_{2}^{\prime} y_{2}\right\rangle^{\prime}=\delta\left(\tau\left(x_{1}\right) x_{2}+\beta \tau\left(y_{1}\right) y_{2}\right),
$$

where $\beta$ is an element in $E$ such that the Hilbert symbol $(-\alpha, \beta)_{F}=-1$. Without loss of generality we assume that $\kappa^{\prime}$ is zero. Then the lattice $L^{\prime}:=v_{1}^{\prime} \mathcal{O}_{E}+v_{2}^{\prime} \mathcal{O}_{E}$ is the only good lattice in $V^{\prime}$ because now $V^{\prime}$ is anisotropic. It is obvious that $L^{\prime *}=v_{1}^{\prime} \mathcal{O}_{E}+v_{2}^{\prime} \mathfrak{p}_{E}^{-1}$. Hence, $G_{L^{\prime}}^{\prime} / G_{L^{\prime}, 0^{+}}^{\prime} \simeq \mathrm{U}_{1}\left(\mathbf{f}_{F}\right) \times \mathrm{U}_{1}\left(\mathbf{f}_{F}\right)$.

Up to equivalence there are three different regular small admissible lattice chains $\mathcal{L}^{\prime}:=\left\{L_{i}^{\prime} \mid i \in \mathbb{Z}\right\}$ in $V^{\prime}$, whose numerical invariants are $(1,0),(1,1),(2,1)$, respectively. The first lattice chain is given by $L_{i}^{\prime}:=L^{\prime} \varpi^{i}$, the second one is given by $L_{i}^{\prime}:=L^{\prime *} \varpi^{i+1}$ and the third one is given by $L_{2 i}^{\prime}=L^{\prime} \varpi^{i}, L_{2 i+1}^{\prime}=L^{\prime *} \varpi^{i}$ for $i \in \mathbb{Z}$.

7.2. Correspondence of representations of depth zero. In this subsection we assume that $\pi$ is of depth zero. Let $L$ be the unique good lattice in $V$. Regarded as a character of $G_{L} / G_{L, 0^{+}} \simeq U(\mathbf{l}) \otimes U\left(\mathbf{l}^{*}\right) \simeq \mathrm{U}_{1}\left(\mathbf{f}_{F}\right) \times \mathrm{U}_{0}\left(\mathbf{f}_{F}\right), \pi$ can be written as $\pi \otimes$ triv. Let $L^{\prime}$ be the unique good lattice in $V^{\prime}$. We know that $G_{L^{\prime}}^{\prime} / G_{L^{\prime}, 0^{+}}^{\prime} \simeq \mathrm{U}_{1}\left(\mathbf{f}_{F}\right) \times \mathrm{U}_{1}\left(\mathbf{f}_{F}\right)$. Suppose that the representation $\pi$ of $U(V)$ occurs in the theta correspondence for the pair $\left(U(V), U\left(V^{\prime}\right)\right)$ and is paired with the irreducible supercuspidal representation $\pi^{\prime}$ of $U\left(V^{\prime}\right)$. Then we also know that $\pi \otimes$ triv is paired with $\tilde{\pi} \otimes$ triv in the correspondence for the finite dual pair $\left(G_{L} / G_{L, 0^{+}}, G_{L^{\prime}}^{\prime} / G_{L^{\prime}, 0^{+}}^{\prime}\right)$. Hence, from subsection 2.6 we know that $\pi$ cannot be the sgn character. Moreover, by Corollary 2.8 we know that $\pi^{\prime}$ is isomorphic to $c$-Ind $G_{G_{L^{\prime}}^{\prime}}^{G^{\prime}}(\tilde{\pi} \otimes$ triv $)$.

7.3. Occurrence. From subsection 7.2 we know that all characters of depth zero except the sgn character occur in the theta correspondence for the dual pair $\left(U(V), U\left(V^{\prime}\right)\right)$. Now we consider the characters of positive depths. Suppose that $V^{\prime \prime}$ is a four-dimensional $\epsilon^{\prime}$-Hermitian space with Witt index one. Since now the pair $\left(U(V), U\left(V^{\prime \prime}\right)\right)$ is in the stable range, the character $\pi$ of $U(V)$ of positive depth occurs in the theta correspondence for the pair $\left(U(V), U\left(V^{\prime \prime}\right)\right)$. Suppose that $\pi$ is paired with an irreducible admissible representation $\left(\pi^{\prime}, \mathcal{V}^{\prime}\right)$ of $U\left(V^{\prime}\right)$. There is an element $w_{0}$ in $B\left(\mathcal{L}, \mathcal{L}^{\prime}, \frac{d}{1}\right)$ for some small admissible lattice chain $\mathcal{L}^{\prime}$ in $V^{\prime}$ such that $\mathfrak{M}\left(w_{0}\right)+\mathfrak{g}_{\mathcal{L},(d / 1)^{+}}$is a minimal $K$-type of $\pi$ and $\mathfrak{M}^{\prime}\left(w_{0}\right)+\mathfrak{g}_{\mathcal{L}^{\prime},(d / 1)^{+}}$is a minimal $K$-type of $\pi^{\prime}$. Let $V^{\prime \prime}=V_{1}^{\prime \prime} \oplus V_{2}^{\prime \prime}$ be the similar decomposition as given in subsection 5.5 where $V_{1}^{\prime \prime}$ is one-dimensional and $V_{2}^{\prime \prime}$ is three-dimensional. Now the Witt index of $V_{2}^{\prime \prime}$ must be one. Let $V_{2}^{\prime \prime}=V_{3}^{\prime \prime} \oplus V_{4}^{\prime \prime}$ be the decomposition of nondegenerate subspaces such that $V_{3}^{\prime \prime}$ is one-dimensional and $V_{4}^{\prime \prime}$ is a hyperplane. Let $V_{5}^{\prime \prime}$ be the direct sum $V_{1}^{\prime \prime} \oplus V_{3}^{\prime \prime}$. So $V^{\prime \prime}=V_{5}^{\prime \prime} \oplus V_{4}^{\prime \prime}$. Clearly the space $V_{5}^{\prime \prime}$ is anisotropic, otherwise it contradicts the assumption that the Witt index of $V^{\prime \prime}$ is one. Let $W^{\prime}=V \otimes V^{\prime \prime}, W_{5}^{\prime}=V \otimes V_{5}^{\prime \prime}$ and $W_{4}^{\prime}=V \otimes V_{4}^{\prime \prime}$. So $W_{4}^{\prime}$ and $W_{5}^{\prime}$ are nondegenerate symplectic subspaces of $W^{\prime}$, and we have the decomposition $W^{\prime}=W_{4}^{\prime} \oplus W_{5}^{\prime}$. As in subsection 5.5 we know that $w_{0}$ is in fact in $W_{5}^{\prime}$. Hence, 
there is a nontrivial map from the Weil representation of $\widetilde{\operatorname{Sp}\left(W_{5}^{\prime}\right)}$ to $\mathcal{V} \otimes \mathcal{V}_{5}^{\prime \prime}$ for some irreducible admissible representation $\left(\pi_{5}^{\prime \prime}, \mathcal{V}_{5}^{\prime \prime}\right)$ of $U\left(V_{5}^{\prime \prime}\right)$. This means that $\pi$ occurs in the theta correspondence for the dual pair $\left(U(V), U\left(V_{5}^{\prime \prime}\right)\right)$. Because now $V_{5}^{\prime \prime}$ is two-dimensional anisotropic, it is isomorphic to the space $V^{\prime}$. Therefore, $\pi$ occurs in the theta correspondence for the dual pair $\left(U(V), U\left(V^{\prime}\right)\right)$. So we have proved the following proposition.

Proposition. Let $\left(U(V), U\left(V^{\prime}\right)\right)$ be the reductive dual pair of unitary groups such that $V$ (resp. $\left.V^{\prime}\right)$ is one-dimensional (resp. anisotropic two-dimensional) over an unramified quadratic extension of $F$. All characters of $U(V)$ except sgn occur in the theta correspondence for the dual pair $\left(U(V), U\left(V^{\prime}\right)\right)$.

7.4. Moment maps. The Lie algebra of $U\left(V^{\prime}\right)$ can be identified with the space of matrices of the form

$$
\left[\begin{array}{cc}
\delta x & -\beta \tau(y) \\
y & \delta z
\end{array}\right]
$$

where $y \in E, x, z \in F$ with respect to the basis $\left\{v_{1}^{\prime}, v_{2}^{\prime}\right\}$. Now the moment maps $\mathfrak{M}, \mathfrak{M}^{\prime}$ are given by

$$
\begin{aligned}
\mathfrak{M}\left(x v_{1} \otimes v_{1}^{\prime}+y v_{1} \otimes v_{2}^{\prime}\right) & =\varpi_{F}^{1-\lambda_{F}} \delta(x \tau(x)+\beta \tau(y) y) \\
\mathfrak{M}^{\prime}\left(x v_{1} \otimes v_{1}^{\prime}+y v_{1} \otimes v_{2}^{\prime}\right) & =-\varpi_{F}^{1-\lambda_{F}} \delta\left[\begin{array}{ll}
x \tau(x) & \beta x \tau(y) \\
y \tau(x) & \beta y \tau(y)
\end{array}\right] .
\end{aligned}
$$

Let $w_{0}$ be as in subsection 5.5. The rank of $\mathfrak{M}^{\prime}\left(w_{0}\right)$ is one. Let $V_{1}^{\prime}\left(\right.$ resp. $\left.V_{2}^{\prime}\right)$ be the eigenspace with respect to a nonzero (resp. zero) eigenvalue. We know that $V_{1}^{\prime}, V_{2}^{\prime}$ are nondegenerate one-dimensional subspaces and $V^{\prime}$ is the orthogonal direct sum of $V_{1}^{\prime}$ and $V_{2}^{\prime}$. By the argument in subsection 5.5 we can check that

$$
L^{\prime}=\left(L^{\prime} \cap V_{1}^{\prime}\right) \oplus\left(L^{\prime} \cap V_{2}^{\prime}\right)
$$

7.5. Correspondence of representations of positive depth. In this subsection we suppose that an irreducible representation $\pi$ of $U(V)$ is of positive depth. We know that the centralizer of $\mathfrak{M}^{\prime}\left(w_{0}\right)$ in $U\left(V^{\prime}\right)$ is $U\left(V_{1}^{\prime}\right) \times U\left(V_{2}^{\prime}\right)$. Let $H$ denote $U\left(V_{1}^{\prime}\right) \times U\left(V_{2}^{\prime}\right)$, and let $J$ be defined as in subsection 5.9. Let $\phi_{\tilde{\pi}}$ be the representation of $H J$ defined as in subsection 5.9. By the same argument in subsections 5.4-5.9 we know that the character $\pi$ of $U(V)$ is paired with the induced representation $c-\operatorname{Ind}_{H J}^{G^{\prime}} \phi_{\tilde{\pi}}$ of $U\left(V^{\prime}\right)$ in the theta correspondence.

\section{Theta correspondence for $\left(\mathrm{U}_{1}(F), \mathrm{U}_{2}(F)\right)$ II}

In this section, we assume that $E$ is a ramified quadratic extension of $F, V$ is one-dimensional and $V^{\prime}$ is two-dimensional anisotropic.

8.1. Lattice chains in $V^{\prime}$. We assume that $E=F(\sqrt{\alpha})$ where $\alpha=\varpi_{F}$. Let $\delta:=\sqrt{\alpha}$; then $\tau(\delta)=-\delta$. We can find a basis $\left\{v_{1}^{\prime}, v_{2}^{\prime}\right\}$ of $V^{\prime}$ such that the form $\langle,\rangle^{\prime}$ on $V^{\prime}$ is given by

$$
\left\langle v_{1}^{\prime} x_{1}+v_{2}^{\prime} y_{1}, v_{1}^{\prime} x_{2}+v_{2}^{\prime} y_{2}\right\rangle^{\prime}=\delta\left(\tau\left(x_{1}\right) x_{2}+\beta \tau\left(y_{1}\right) y_{2}\right),
$$

where $\beta$ is an element in $E$ such that the Hilbert symbol $\left(-\varpi_{F}, \beta\right)_{F}=-1$. We assume that $\kappa^{\prime}$ is zero. Then the lattice $L^{\prime}:=v_{1}^{\prime} \mathcal{O}_{E}+v_{2}^{\prime} \mathcal{O}_{E}$ is the only good lattice in $V^{\prime}$. It is easy to see that $G_{L^{\prime}}^{\prime} / G_{L^{\prime}, 0^{+}}^{\prime}$ is isomorphic to $\mathrm{O}_{2}\left(\mathbf{f}_{F}\right) \times \operatorname{Sp}_{0}\left(\mathbf{f}_{F}\right)$.

Up to equivalence there is only one regular small admissible lattice chain in $V^{\prime}$, whose numerical invariant is $(1,1)$ and is generated by $\left\{L^{\prime}\right\}$. 
8.2. Correspondence of representations of depth zero. In this subsection let $\pi$ be a character of $U(V)$ of depth zero. So we know that $\pi$ is either triv or sgn. Suppose that $\pi$ occurs in the theta correspondence for the pair $\left(U(V), U\left(V^{\prime}\right)\right)$ and is paired with the irreducible supercuspidal representation $\pi^{\prime}$ of $U\left(V^{\prime}\right)$. Let $L, L^{\prime}$ be the good lattices in $V, V^{\prime}$, respectively. Then $G_{L} / G_{L, 0^{+}}$is isomorphic to $\mathrm{O}_{1}\left(\mathbf{f}_{F}\right) \times \operatorname{Sp}_{0}\left(\mathbf{f}_{F}\right)$ and $G_{L^{\prime}}^{\prime} / G_{L^{\prime}, 0^{+}}^{\prime}$ is isomorphic to $\operatorname{Sp}_{0}\left(\mathbf{f}_{F}\right) \times \mathrm{O}_{2}\left(\mathbf{f}_{F}\right)$. Therefore, the dual pair $\left(G_{L} / G_{L, 0^{+}}, G_{L^{\prime}}^{\prime} / G_{L^{\prime}, 0^{+}}^{\prime}\right)$ is $\left(\mathrm{O}_{1}\left(\mathbf{f}_{F}\right) \times \operatorname{Sp}_{0}\left(\mathbf{f}_{F}\right), \operatorname{Sp}_{0}\left(\mathbf{f}_{F}\right) \times \mathrm{O}_{2}\left(\mathbf{f}_{F}\right)\right)$. Hence, $\pi$ has to be the trivial representation of $U(V)$, and it is paired with the trivial representation of $U\left(V^{\prime}\right)$.

8.3. Occurrence. We know that $U(V)$ has two characters of depth zero, which are triv and sgn. From the result in subsection 8.2 we know that triv occurs in the correspondence for the pair $\left(U(V), U\left(V^{\prime}\right)\right)$ but sgn does not. Next we consider the occurrence of representations of positive depths. Clearly Proposition 7.3 still holds when $E$ is a ramified extension of $F$. Hence, we have the following proposition.

Proposition. Let $\left(U(V), U\left(V^{\prime}\right)\right)$ be the reductive dual pair of unitary groups such that $V$ (resp. $\left.V^{\prime}\right)$ is one-dimensional (resp. anisotropic two-dimensional) over a ramified quadratic extension of $F$. All characters of $U(V)$ except $\mathrm{sgn}$ occur in the theta correspondence for the dual pair $\left(U(V), U\left(V^{\prime}\right)\right)$.

8.4. Correspondence of representations of positive depths. The correspondence of representations of positive depths is the same as in the case for unramified $E$ in subsection 7.5 .

\section{Theta CORRespondence For $\left(\mathrm{U}_{1}(F), \mathrm{U}_{1,2}(F)\right) \mathrm{I}$}

In this section, we assume that $E$ is an unramified quadratic extension of $F, V$ is one-dimensional and $V^{\prime}$ is three-dimensional.

9.1. Occurrence. Let $V^{\prime \prime}$ be the $\epsilon^{\prime}$-Hermitian space which is the preceding term of $V^{\prime}$ in the Witt series. So $V^{\prime \prime}$ is one-dimensional. Let $\pi$ be a character of $G:=U(V)$. Because now we want investigate the correspondence of supercuspidal representations, we assume that $\pi$ does not occur in the theta correspondence for the dual pair $\left(U(V), U\left(V^{\prime \prime}\right)\right)$. Then $\pi$ must occur in the theta correspondence for the dual pair $\left(U(V), U\left(V^{\prime}\right)\right)$ because the pair $\left(U(V), U\left(V^{\prime}\right)\right)$ is in the stable range. Let $\pi^{\prime}$ be the irreducible supercuspidal representation of $G^{\prime}:=U\left(V^{\prime}\right)$ paired with $\pi$.

9.2. Lattice chains in $V^{\prime}$. Let $\delta$ be an element in $\mathcal{O}_{E}^{*}$ such that $\tau(\delta)=-\delta$. We can find a basis $\left\{v_{1}^{\prime}, v_{2}^{\prime}, v_{3}^{\prime}\right\}$ of $V^{\prime}$ such that the form $\langle,\rangle^{\prime}$ on $V^{\prime}$ is given by

$$
\left\langle v_{1}^{\prime} x_{1}+v_{2}^{\prime} y_{1}+v_{3}^{\prime} z_{1}, v_{1}^{\prime} x_{2}+v_{2}^{\prime} y_{2}+v_{3}^{\prime} z_{2}\right\rangle^{\prime}=\tau\left(x_{1}\right) z_{2}+\delta \tau\left(y_{1}\right) y_{2}-\tau\left(z_{1}\right) x_{2} .
$$

Without loss of generality we assume that $\kappa^{\prime}$ is zero. Now the lattices

$$
\begin{aligned}
& M_{0}^{\prime}:=v_{1}^{\prime} \mathcal{O}_{E}+v_{2}^{\prime} \mathcal{O}_{E}+v_{3}^{\prime} \mathcal{O}_{E} \\
& M_{1}^{\prime}:=v_{1}^{\prime} \mathfrak{p}_{E}+v_{2}^{\prime} \mathcal{O}_{E}+v_{3}^{\prime} \mathcal{O}_{E}
\end{aligned}
$$

are the only good lattices in $V^{\prime}$ up to equivalence. Let $L^{\prime}$ be a good lattice in $V^{\prime}$. Then we know that $G_{L^{\prime}}^{\prime} / G_{L^{\prime}, 0^{+}}^{\prime}$ is isomorphic to $\mathrm{U}_{3}\left(\mathbf{f}_{F}\right) \times \mathrm{U}_{0}\left(\mathbf{f}_{F}\right)$ if $L^{\prime}=M_{0}^{\prime}$ and is isomorphic to $\mathrm{U}_{1}\left(\mathbf{f}_{F}\right) \times \mathrm{U}_{2}\left(\mathbf{f}_{F}\right)$ if $L^{\prime}=M_{1}^{\prime}$. 
There are seven regular small admissible lattice chains in $V^{\prime}$. The numerical invariants of them are

$$
\begin{cases}(1,0), & \text { if } L_{i}^{\prime}:=M_{0}^{\prime} \varpi^{i} ; \\ (1,0), & \text { if } L_{i}^{\prime}:=M_{1}^{\prime} \varpi^{i} ; \\ (1,1), & \text { if } L_{i}^{\prime}:=M_{1}^{\prime *} \varpi^{i+1} ; \\ (2,0), & \text { if } L_{2 i}^{\prime}:=M_{0}^{\prime} \varpi^{i}, L_{2 i+1}^{\prime}:=M_{1}^{\prime} \varpi^{i} \\ (2,0), & \text { if } L_{2 i}^{\prime}:=M_{0}^{\prime} \varpi^{i}, L_{2 i+1}^{\prime}:=M_{1}^{\prime *} \varpi^{i+1} ; \\ (2,1), & \text { if } L_{2 i}^{\prime}:=M_{1}^{\prime} \varpi^{i}, L_{2 i+1}^{\prime}:=M_{1}^{\prime *} \varpi^{i+1} ; \\ (3,0), & \text { if } L_{3 i}^{\prime}:=M_{1}^{\prime} \varpi^{i}, L_{3 i+1}^{\prime}:=M_{1}^{\prime} \varpi^{i}, L_{3 i+2}^{\prime}:=M_{1}^{\prime *} \varpi^{i+1}\end{cases}
$$

9.3. Correspondence of representations of depth zero. In this subsection we assume that $\pi$ is of depth zero. Let $L$ be the unique good lattice in $V$. We know that $G_{L} / G_{L, 0^{+}} \simeq \mathrm{U}_{1}\left(\mathbf{f}_{F}\right) \times \mathrm{U}_{0}\left(\mathbf{f}_{F}\right)$.

Suppose that $\lambda_{F}$ is odd. Then $\pi$, regarded as a representation of $G_{L} / G_{L, 0^{+}} \simeq$ $\mathrm{U}_{1}\left(\mathbf{f}_{F}\right) \times \mathrm{U}_{0}\left(\mathbf{f}_{F}\right)$, is $\operatorname{sgn} \otimes$ triv. Let $L^{\prime}$ be the good lattice in $V^{\prime}$ such that $G_{L^{\prime}}^{\prime} / G_{L^{\prime}, 0^{+}}^{\prime}$ $\simeq \mathrm{U}_{3}\left(\mathbf{f}_{F}\right) \times \mathrm{U}_{0}\left(\mathbf{f}_{F}\right)$. Let $\eta^{\prime}$ be the $\left(q^{2}-q\right)$-dimensional irreducible cuspidal representation of $\mathrm{U}_{3}\left(\mathbf{f}_{F}\right)$ paired with $\pi$. From subsection 2.8 we know that $\pi^{\prime}$ has a minimal $K$-type $\left(G_{L^{\prime}}^{\prime}, \eta^{\prime} \otimes\right.$ triv), where triv is the trivial representation of the trivial group $\mathrm{U}_{0}\left(\mathbf{f}_{F}\right)$. Hence, $\pi^{\prime}$ is isomorphic to $c$-Ind $\operatorname{In}_{L^{\prime}}^{G^{\prime}}\left(\eta^{\prime} \otimes\right.$ triv $)$.

Next we suppose that $\lambda_{F}$ is even. Then $\pi$, regarded as a representation of $G_{L} / G_{L, 0^{+}} \simeq \mathrm{U}_{1}\left(\mathbf{f}_{F}\right) \times \mathrm{U}_{0}\left(\mathbf{f}_{F}\right)$, is $\pi \otimes$ triv, and $\pi$ cannot be the sgn character. Let $L^{\prime}$ be the good lattice in $V^{\prime}$ such that $G_{L^{\prime}}^{\prime} / G_{L^{\prime}, 0^{+}}^{\prime} \simeq \mathrm{U}_{2}\left(\mathbf{f}_{F}\right) \times \mathrm{U}_{1}\left(\mathbf{f}_{F}\right)$. Let $\eta^{\prime}$ be the $(q-1)$-dimensional irreducible cuspidal representation of $\mathrm{U}_{2}\left(\mathbf{f}_{F}\right)$ paired with $\pi$. From subsection 2.8 we know that $\pi^{\prime}$ has a minimal $K$-type $\left(G_{L^{\prime}}^{\prime}, \eta^{\prime} \otimes\right.$ triv $)$. Hence, $\pi^{\prime}$ is isomorphic to $c$ - $\operatorname{Ind}_{G_{L^{\prime}}^{\prime}}^{G^{\prime}}\left(\eta^{\prime} \otimes\right.$ triv $)$.

9.4. Moment maps. The moment maps $\mathfrak{M}, \mathfrak{M}^{\prime}$ are given by

$$
\begin{aligned}
\mathfrak{M}\left(x v_{1} \otimes v_{1}^{\prime}+y v_{1} \otimes v_{2}^{\prime}+z v_{1} \otimes v_{3}^{\prime}\right) & =\varpi_{F}^{1-\lambda_{F}}(x \tau(z)+\delta \tau(y) y-\tau(x) z) \\
\mathfrak{M}^{\prime}\left(x v_{1} \otimes v_{1}^{\prime}+y v_{1} \otimes v_{2}^{\prime}+z v_{1} \otimes v_{3}^{\prime}\right) & =-\varpi_{F}^{1-\lambda_{F}} \delta\left[\begin{array}{lll}
-x \tau(z) & \delta x \tau(y) & x \tau(x) \\
-y \tau(z) & \delta y \tau(y) & y \tau(x) \\
-z \tau(z) & \delta z \tau(y) & z \tau(x)
\end{array}\right] .
\end{aligned}
$$

Let $w_{0}$ be as in subsection 5.5. The rank of $\mathfrak{M}^{\prime}\left(w_{0}\right)$ is one. Let $V_{1}^{\prime}$ (resp. $\left.V_{2}^{\prime}\right)$ be the eigenspace with respect to a nonzero (resp. zero) eigenvalue. We know that $V_{1}^{\prime}$ (resp. $V_{2}^{\prime}$ ) is a one-dimensional (resp. two-dimensional) subspace of $V$. Moreover, $V_{1}^{\prime}, V_{2}^{\prime}$ are nondegenerate subspaces, and $V^{\prime}$ is the orthogonal direct sum of $V_{1}^{\prime}$ and $V_{2}^{\prime}$. By the argument in subsection 5.5 we can check that

$$
L^{\prime}=\left(L^{\prime} \cap V_{1}^{\prime}\right) \oplus\left(L^{\prime} \cap V_{2}^{\prime}\right) .
$$

9.5. Decomposition of $V^{\prime}$. Now suppose that $\pi$ is a character of $U(V)$ of positive depth which first occurs in the theta correspondence for the dual pair $\left(U(V), U\left(V^{\prime}\right)\right)$. As in subsection 5.5, $V^{\prime}$ has a decomposition $V_{1}^{\prime} \oplus V_{2}^{\prime}$ as a direct sum of two nondegenerate subspaces. Now $V_{1}^{\prime}$ is one-dimensional and $V_{2}^{\prime}$ is two-dimensional.

Lemma. The restriction of the form $\langle,\rangle^{\prime}$ on $V_{2}^{\prime}$ is anisotropic.

Proof. Let $\langle,\rangle_{2}^{\prime}$ denote the restriction of $\langle,\rangle^{\prime}$ on $V_{2}^{\prime}$. Suppose that the form $\langle,\rangle_{2}^{\prime}$ is not anisotropic. Then $V_{2}^{\prime}$ is a hyperplane. Hence, $V_{1}^{\prime}$ is the preceding term of $V^{\prime}$ in the Witt series. 
Hence, $\pi$ occurs in the theta correspondence for the dual pair $\left(U(V), U\left(V_{1}^{\prime}\right)\right)$. This contradicts the assumption that $\pi$ first occurs in the theta correspondence for the dual pair $\left(U(V), U\left(V^{\prime}\right)\right)$. Therefore, the form $\langle,\rangle_{2}^{\prime}$ is anisotropic.

9.6. Correspondence of representations of positive depths. Keep the assumption that $\pi$ is a character of $U(V)$ of positive depth which first occurs in the theta correspondence for the dual pair $\left(U(V), U\left(V^{\prime}\right)\right)$. Let $w_{0}$ be as in subsection 5.5. The centralizer of $\mathfrak{M}^{\prime}\left(w_{0}\right)$ in $U\left(V^{\prime}\right)$ is $U\left(V_{1}^{\prime}\right) \times U\left(V_{2}^{\prime}\right)$, which is anisotropic by Lemma 9.5. Hence, the arguments in subsections 5.5-5.9 still work in the present situation. So we conclude that the character $\pi$ of $U(V)$ is paired with the induced representation $c-\operatorname{Ind}_{H J}^{G^{\prime}} \phi_{\tilde{\pi}}$ of $U\left(V^{\prime}\right)$ in the theta correspondence.

\section{Theta correspondence For $\left(\mathrm{U}_{1}(F), \mathrm{U}_{1,2}(F)\right)$ II}

In this section, we assume that $E$ is a ramified quadratic extension of $F, V$ is one-dimensional and $V^{\prime}$ is three-dimensional.

10.1. Occurrence. Let $V^{\prime \prime}$ be given as in subsection 9.1. We assume that the character $\pi$ of $U(V)$ does not occur in the theta correspondence for the dual pair $\left(U(V), U\left(V^{\prime \prime}\right)\right)$. Let $\pi^{\prime}$ be the irreducible supercuspidal representation of $U\left(V^{\prime}\right)$ paired with $\pi$ in the correspondence for the pair $\left(U(V), U\left(V^{\prime}\right)\right)$.

10.2. Lattice chains in $V^{\prime}$. There are two good lattices in $V^{\prime}$. We know that $G_{L^{\prime}}^{\prime} / G_{L^{\prime}, 0^{+}}^{\prime}$ is isomorphic to either $\mathrm{O}_{1}\left(\mathbf{f}_{F}\right) \times \operatorname{Sp}_{2}\left(\mathbf{f}_{F}\right)$ or $\mathrm{O}_{1,2}\left(\mathbf{f}_{F}\right) \times \operatorname{Sp}_{0}\left(\mathbf{f}_{F}\right)$ when $L^{\prime}$ is a good lattice in $V^{\prime}$.

Let $M_{1}^{\prime} \subset M_{0}^{\prime}$ be the two good lattices in $V^{\prime}$. There are seven regular small admissible lattice chains in $V^{\prime}$, which are generated by $\left\{M_{0}^{\prime}\right\},\left\{M_{0}^{\prime *}\right\}\left\{M_{0}^{\prime}, M_{0}^{\prime *}\right\}$, $\left\{M_{1}^{\prime}\right\},\left\{M_{1}^{\prime}, M_{0}^{\prime}\right\},\left\{M_{1}^{\prime}, M_{0}^{\prime *}\right\}$ and $\left\{M_{1}^{\prime}, M_{0}^{\prime}, M_{0}^{\prime *}\right\}$. Their numerical invariants are $(1,0),(1,1),(2,1),(1,1),(2,0),(2,1)$ and $(3,1)$, respectively.

10.3. Correspondence of representations of depth zero. In this subsection we assume that $\pi$ is of depth zero. Let $L$ be the unique good lattice in $V$. We know that $G_{L} / G_{L, 0^{+}} \simeq \mathrm{O}_{1}\left(\mathbf{f}_{F}\right) \times \operatorname{Sp}_{0}\left(\mathbf{f}_{F}\right)$. Because we assume that $\pi$ does not occur in the theta correspondence for the dual pair $\left(U(V), U\left(V^{\prime \prime}\right)\right)$, we know that $\pi$, regarded as a representation of $G_{L} / G_{L, 0^{+}} \simeq \mathrm{O}_{1}\left(\mathbf{f}_{F}\right) \times \operatorname{Sp}_{0}\left(\mathbf{f}_{F}\right)$, is $\operatorname{sgn} \otimes$ triv. The representation $\operatorname{sgn} \otimes$ triv is paired with the representation $\eta^{\prime} \otimes$ triv of $\operatorname{Sp}_{2}\left(\mathbf{f}_{F}\right) \times \mathrm{O}_{1}\left(\mathbf{f}_{F}\right)$ when it first occurs, where $\eta^{\prime}$ is a $\frac{q-1}{2}$-dimensional irreducible cuspidal representation of $\operatorname{Sp}_{2}\left(\mathbf{f}_{F}\right)$. Then we know that the representation $\pi^{\prime}$ is isomorphic to $c-\operatorname{Ind}_{G_{L^{\prime}}^{\prime}}^{G^{\prime}}\left(\eta^{\prime} \otimes\right.$ triv), where $L^{\prime}$ is a good lattice in $V^{\prime}$ such that $G_{L^{\prime}}^{\prime} / G_{L^{\prime}, 0^{+}}^{\prime} \simeq \operatorname{Sp}_{2}\left(\mathbf{f}_{F}\right) \times \mathrm{O}_{1}\left(\mathbf{f}_{F}\right)$.

10.4. Correspondence of representations of positive depths. The explicit correspondence of representations of positive depths is the same as in the case for unramified $E$ in subsection 9.6.

\section{Theta correspondence for $\left(\mathrm{U}_{1}(F), \mathrm{U}_{1,3}(F)\right) \mathrm{I}$}

In this section, we assume that $E$ is an unramified quadratic extension of $F, V$ is one-dimensional and $V^{\prime}$ is four-dimensional with Witt index one. 
11.1. Correspondence of representations of depth zero. From the result in section 7 we know that the only representation of $U(V)$ which does not occur in the correspondence for the pair $\left(U(V), U\left(V^{\prime \prime}\right)\right)$ is the sgn character, where $V^{\prime \prime}$ is the two-dimensional anisotropic $\epsilon^{\prime}$-Hermitian space. So now we assume that $\pi$ is the sgn character of $U(V)$. Let $L$ be the unique good lattice in $V$. We know that $G_{L} / G_{L, 0^{+}} \simeq \mathrm{U}_{1}\left(\mathbf{f}_{F}\right) \times \mathrm{U}_{0}\left(\mathbf{f}_{F}\right)$. We also know that there are two (equivalence classes of) good lattices $L^{\prime}$ in $V^{\prime}$, and $G_{L^{\prime}}^{\prime} / G_{L^{\prime}, 0^{+}}^{\prime}$ is isomorphic to either $\mathrm{U}_{1}\left(\mathbf{f}_{F}\right) \times \mathrm{U}_{3}\left(\mathbf{f}_{F}\right)$ or $\mathrm{U}_{3}\left(\mathbf{f}_{F}\right) \times \mathrm{U}_{1}\left(\mathbf{f}_{F}\right)$. Now $\pi$, regarded as a representation of $G_{L} / G_{L, 0^{+}} \simeq \mathrm{U}_{1}\left(\mathbf{f}_{F}\right) \times$ $\mathrm{U}_{0}\left(\mathbf{f}_{F}\right)$, is $\operatorname{sgn} \otimes$ triv. The representation $\operatorname{sgn} \otimes$ triv is paired with the representation $\eta^{\prime} \otimes$ triv of $\mathrm{U}_{3}\left(\mathbf{f}_{F}\right) \times \mathrm{U}_{1}\left(\mathbf{f}_{F}\right)$ when it first occurs, where $\eta^{\prime}$ is a $\left(q^{2}-q\right)$-dimensional irreducible cuspidal representation of $\mathrm{U}_{3}\left(\mathbf{f}_{F}\right)$ ( $c f$. How). Then we know that the representation $\pi^{\prime}$ is isomorphic to $c$-Ind $\operatorname{Ind}_{G^{\prime}{ }^{\prime}}^{G^{\prime}}\left(\eta^{\prime} \otimes\right.$ triv), where $L^{\prime}$ is a good lattice in $V^{\prime}$ such that $G_{L^{\prime}}^{\prime} / G_{L^{\prime}, 0^{+}}^{\prime} \simeq \mathrm{U}_{3}\left(\mathbf{f}_{F}\right) \times \mathrm{U}_{1}\left(\mathbf{f}_{F}\right)$.

\section{Theta Correspondence For $\left(\mathrm{U}_{1}(F), \mathrm{U}_{1,3}(F)\right)$ II}

In this section, we assume that $E$ is a ramified quadratic extension of $F, V$ is one-dimensional and $V^{\prime}$ is four-dimensional with Witt index one.

12.1. Correspondence of representations of depth zero. As in subsection 11.1 we let $\pi$ be the sgn character of $U(V)$. Let $L$ be the unique good in $V$. We know that $G_{L} / G_{L, 0^{+}} \simeq \mathrm{O}_{1}\left(\mathbf{f}_{F}\right) \times \operatorname{Sp}_{0}\left(\mathbf{f}_{F}\right)$. There are two (equivalence classes of good lattices $L^{\prime}$ in $V^{\prime}$. We know that $G_{L^{\prime}}^{\prime} / G_{L^{\prime}, 0^{+}}^{\prime}$ is isomorphic to either $\mathrm{O}_{2}\left(\mathbf{f}_{F}\right) \times \operatorname{Sp}_{2}\left(\mathbf{f}_{F}\right)$ or $\mathrm{O}_{1,3}\left(\mathbf{f}_{F}\right) \times \operatorname{Sp}_{0}\left(\mathbf{f}_{F}\right)$. Now $\pi$, regarded as a representation of $G_{L} / G_{L, 0^{+}} \simeq \mathrm{O}_{1}\left(\mathbf{f}_{F}\right) \times$ $\operatorname{Sp}_{0}\left(\mathbf{f}_{F}\right)$, is sgn $\otimes$ triv. The representation $\operatorname{sgn} \otimes \operatorname{triv}$ of $\mathrm{O}_{1}\left(\mathbf{f}_{F}\right) \times \operatorname{Sp}_{0}\left(\mathbf{f}_{F}\right)$ is paired with the representation $\eta^{\prime} \otimes$ triv of $\operatorname{Sp}_{2}\left(\mathbf{f}_{F}\right) \times \mathrm{O}_{2}\left(\mathbf{f}_{F}\right)$ when it first occurs, where $\eta^{\prime}$ is a $\frac{q-1}{2}$-dimensional irreducible cuspidal representation of $\operatorname{Sp}_{2}\left(\mathbf{f}_{F}\right)$. Then we know that the representation $\pi^{\prime}$ is isomorphic to $c$ - $\operatorname{Ind}_{G_{L^{\prime}}^{\prime}}^{G^{\prime}}\left(\eta^{\prime} \otimes\right.$ triv), where $L^{\prime}$ is the good lattice in $V^{\prime}$ such that $G_{L^{\prime}}^{\prime} / G_{L^{\prime}, 0^{+}}^{\prime} \simeq \operatorname{Sp}_{2}\left(\mathbf{f}_{F}\right) \times \mathrm{O}_{2}\left(\mathbf{f}_{F}\right)$.

\section{Preservation PRinciple}

13.1. Preservation principle. Let $\varepsilon_{E / F}$ denote the quadratic character of $E$ over $F$. Let $V$ be an $\epsilon$-Hermitian space over $E$, and let $V^{\prime \pm}$ be the $\epsilon^{\prime}$-Hermitian space given by the condition

$$
\varepsilon_{E / F}\left((-1)^{\frac{m^{ \pm}\left(m^{ \pm}-1\right)}{2}} \operatorname{det}\left(V^{\prime \pm}\right)\right)= \pm 1,
$$

where $m^{ \pm}$is the dimension of $V^{\prime \pm}$. Let $\pi$ be an irreducible admissible representation of $U(V)$, and let sgn be the sign character of $U(V)$. Let $m_{0}^{+}(\pi)$ (resp. $\left.m_{0}^{-}(\pi \otimes \operatorname{sgn})\right)$ denote the dimension of $V^{\prime+}\left(\right.$ resp. $\left.V^{\prime-}\right)$ such that $\pi($ resp. $\pi \otimes \operatorname{sgn})$ first occurs in the theta correspondence for the reductive dual pair $\left(U(V), U\left(V^{\prime+}\right)\right)$ (resp. $\left.\left(U(V), U\left(V^{\prime+}\right)\right)\right)$. It is conjectured in HKS96 (see also Pan02b) that

$$
m_{0}^{+}(\pi)+m_{0}^{-}(\pi \otimes \operatorname{sgn})=2 \operatorname{dim}(V)+2 .
$$

This conjecture is the so-called preservation principle of the theta correspondence. This conjecture is proved to be true for all supercuspidal representations $\pi$ in HKS96] when the dimensions of $V$ and $V^{\prime}$ are of the same parity. Then the result is extended in Pan02b] for all depth zero supercuspidal representations $\pi$ without the restriction on the parity of dimensions of $V$ and $V^{\prime}$. 
13.2. For $V$ one-dimensional. In this subsection we want to verify the preservation principle when $V$ is one-dimensional. Now we assume that $V$ is one-dimensional and $\pi$ is a character of $U(V)$.

First we suppose that the dimension of $V$ and the dimension of $V^{\prime}$ have the same parity. From the result in sections $3,4,9,10$ we know that

$$
m_{0}^{+}(\pi)= \begin{cases}1, & \text { if } \pi=\text { triv or the depth of } \pi \text { is positive even; } \\ 3, & \text { otherwise, } \\ m_{0}^{-}(\pi) & \text { if } \pi \neq \text { sgn or the depth of } \pi \text { is odd } \\ 3, & \text { otherwise }\end{cases}
$$

Then we suppose that the dimension of $V$ and the dimension of $V^{\prime}$ have the opposite parity. From the result in sections $5,6,7,8,11,12$ we know that

$$
\begin{aligned}
& m_{0}^{+}(\pi)= \begin{cases}0, & \text { if } \pi=\text { triv } ; \\
2, & \text { if } \pi \neq \text { triv },\end{cases} \\
& m_{0}^{-}(\pi)= \begin{cases}2, & \text { if } \pi \neq \operatorname{sgn} ; \\
4, & \text { if } \pi=\operatorname{sgn} .\end{cases}
\end{aligned}
$$

Therefore, the preservation principle (13.1.2) is verified when $V$ is one-dimensional.

\section{REFERENCES}

[Ad198] J. Adler, Refined anisotropic K-types and supercuspidal representations, Pacific J. Math. 185 (1998), 1-32. MR.1653184 (2000f:22019)

[HKS96] M. Harris, S. Kudla, and W. Sweet, Theta dichotomy for unitary groups, J. Amer. Math. Soc. 9 (1996), 941-1004. MR1327161 (96m:11041)

[How] R. Howe, Invariant theory and duality for classical groups over finite fields with applications to their singular representation theory, preprint.

[How79] $\quad \theta$-series and invariant theory, Automorphic forms, representations and Lfunctions, Proc. Sympos. Pure Math., vol. 33, 1979, pp. 275-285. MR 0546602 (81f:22034)

[Kud86] S. Kudla, On the local theta correspondence, Invent. Math. 83 (1986), 229-255. MR0818351 (87e:22037)

[Kud94] S. Kudla, Splitting metaplectic covers of dual reductive pairs, Israel J. Math. 87 (1994), 361-401. MR1286835 (95h:22019)

[Moe87] C. Moen, The dual pair $(U(3), U(1))$ over a p-adic field, Pacific J. Math. 127 (1987), 141-154. MR0876022 (88e:22025)

[Moe93] , The dual pair $(U(1), U(1))$ over a p-adic field, Pacific J. Math. 158 (1993), 365-386. MR1206444 (94a:22036)

[MP94] A. Moy and G. Prasad, Unrefined minimal K-types for p-adic groups, Invent. Math. 116 (1994), 393-408. MR.1253198 (95f:22023)

[MP96] U Jacquet functors and unrefined minimal K-types, Comment Math. Helv. 71 (1996), 98-121. MR1371680 (97c:22021)

[MVW87] C. Møeglin, M.-F. Vigneras, and J.-L. Waldspurger, Correspondances de Howe sur un corps p-adiques, Lecture Notes in Math., vol. 1291, Springer-Verlag, Berlin, Heidelberg, New York, 1987. MR1041060 (91f:11040)

[Pan01] S.-Y. Pan, Splittings of metaplectic covers of some reductive dual pairs, Pacific J. Math. 199 (2001), 163-226. MR.1847153 (2002g:22027)

[Pan02a] , Depth preservation in local theta correspondence, Duke Math. J. 113 (2002), 531-592. MR1909608 (2003f:22024)

[Pan02b] Local theta correspondence of representations of depth zero and theta dichotomy, J. Math. Soc. Japan 54 (2002), 793-845. MR1921088 (2003f:11070)

[Pan03] L Local theta correspondence and minimal k-types of positive depth, Israel J. Math. 138 (2003), 317-352. MR2031962 (2005h:22029)

[ST69] J. Shalika and S. Tanaka, On an explicit construction of a certain class of automorphic forms, Amer. J. Math. 91 (1969), 1049-1076. MR0291087 (45:181) 
[Wa190] J.-L. Waldspurger, Demonstration d'une conjecture de duality de Howe dans le case $p$-adiques, $p \neq 2$, Festschrift in honor of I. Piatetski-Shapiro, Israel Math. Conf. Proc., vol. 2, 1990, pp. 267-324. MR.1159105 (93h:22035)

[Yan98] T. Yang, Eigenfunctions of the Weil representation of unitary group of one variable, Trans. Amer. Math. Soc. 350 (1998), 2393-2407. MR.1407714(98h:11052)

[Yu98] J.-K. Yu, Unrefined minimal K-types for p-adic classical groups, preprint, 1998.

National Center for Theoretical Sciences and Department of Mathematics, National Cheng Kung University, Tainan City 701, Tainan

E-mail address: sypan@mail.ncku.edu.tw

Current address: National Center for Theoretical Sciences and Department of Mathematics, National Tsing Hua University, Hsinchu 300, Taiwan

E-mail address: sypan@math.nthu.edu.tw 